\title{
How to Construct Quantum Random Functions
}

\author{
MARK ZHANDRY \\ Stanford University, USA \\ mzhandry@stanford.edu
}

\begin{abstract}
In the presence of a quantum adversary, there are two possible definitions of security for a pseudorandom function. The first, which we call standard-security, allows the adversary to be quantum, but requires queries to the function to be classical. The second, quantum-security, allows the adversary to query the function on a quantum superposition of inputs, thereby giving the adversary a superposition of the values of the function at many inputs at once. Existing techniques for proving the security of pseudorandom functions fail when the adversary can make quantum queries. We give the first quantum-security proofs for pseudorandom functions by showing that some classical constructions of pseudorandom functions are quantum-secure. Namely, we show that the standard constructions of pseudorandom functions from pseudorandom generators or pseudorandom synthesizers are secure, even when the adversary can make quantum queries. We also show that a direct construction from lattices is quantum-secure. To prove security, we develop new tools to prove the indistinguishability of distributions under quantum queries.

In light of these positive results, one might hope that all standard-secure pseudorandom functions are quantum-secure. To the contrary, we show a separation: under the assumption that standard-secure pseudorandom functions exist, there are pseudorandom functions secure against quantum adversaries making classical queries, but insecure once the adversary can make quantum queries.
\end{abstract}

Keywords: Quantum, Pseudorandom Function

\section{Introduction}

In their seminal paper, Goldreich, Goldwasser, and Micali [GGM86] answer the question of how to construct a function that looks random to classical adversaries. Specifically, they define a pseudorandom function (PRF) as a function PRF with the following property: no efficient classical algorithm, when given oracle access to PRF, can distinguish PRF from a truly random function. They then construct such a pseudorandom function from pseudorandom generators. Since then, pseudorandom functions have also been built from pseudorandom synthesizers [NR95], as well as directly from hard problems [NR97, NRR00, DY05, LW09, BMR10, BPR11]. Pseudorandom functions have become an important tool in cryptography: for example, they are used in the construction of identification protocols, block ciphers, and message authentication codes.

To define pseudorandom functions in the presence of a quantum adversary, two approaches are possible. The first is what we call standard-security: the quantum adversary can only make classical queries to the function, but all the computation between the queries may be quantum. The second, which we call quantum-security, allows the adversary to make quantum queries to the function. 
That is, the adversary can send a quantum superposition of inputs to the function, and receives a superposition of the corresponding outputs in return. We call pseudorandom functions that are secure against quantum queries Quantum Pseudorandom Functions, or QPRFs. Constructing secure QPRFs will be the focus of this paper.

Quantum-secure pseudorandom functions (QPRFs) have several applications. Whenever a pseudorandom function is used in the presence of a quantum adversary, security against quantum queries captures a wider class of attacks. Thus, the conservative approach to cryptosystem design would dictate using a quantum-secure pseudorandom function. Further, in any instance where a pseudorandom function might be evaluated on a superposition, quantum-security is required. For example, classically, pseudorandom functions work as message authentication codes (MACs). In the quantum world, however, it may be possible for a quantum adversary to query the MAC on a superposition of messages, thus necessitating the use of a quantum-secure pseudorandom function.

Lastly, quantum-secure pseudorandom functions can be used to simulate quantum-accessible random oracles $\left[\mathrm{BDF}^{+} 11\right]$. Unlike the classical setting, where a random oracle can be simulated on the fly, simulating a quantum-accessible random oracle requires defining the entire function up front before any queries are made. Zhandry [Zha12] observes that if the number of queries is a-priori bounded by $q, 2 q$-wise independent functions are sufficient. However, whenever the number of quantum queries is not known in advance, quantum-secure pseudorandom functions seem necessary for simulating quantum-accessible random oracles.

\subsection{Proving Quantum Security}

Goldreich, Goldwasser, and Micali show how to build a pseudorandom function PRF from any length-doubling pseudorandom generator $G$. This construction is known as the GGM construction. Pseudorandom generators can, in turn, be built from any one-way function, as shown by Håstad et al. [HILL99]. The security proof of Håstad et al. is entirely black-box, meaning that it carries over to the quantum setting immediately if the underlying one-way function is secure against quantum adversaries. However, we will now see that the classical proof of security for the GGM construction does not hold in the quantum world.

At a high level, implicit in the GGM construction is a binary tree of depth $n$, where each leaf corresponds to an input/output pair of PRF. To evaluate PRF, we start at the root, and follow the path from root to the leaf corresponding to the input. The security proof consists of two hybrid arguments: the first across levels of the tree, and the second across the nodes in a particular level. The first step has only polynomially many hybrids since the tree's depth is a polynomial. For the second step, a classical adversary can only query PRF on polynomially many points, so the paths used to evaluate PRF only visit polynomially many nodes in each level. Therefore, we only need polynomially many hybrids for the second hybrid argument. This allows any adversary $A$ that breaks the security of PRF with probability $\epsilon$ to be converted into an adversary $B$ that breaks the security of $G$ with probability only polynomially smaller that $\epsilon$.

In the quantum setting, $A$ may query PRF on a superposition of all inputs, so the response to even a single query could require visiting all nodes in the tree. Each level of the tree may have exponentially many nodes, so the second hybrid argument above would need exponentially many hybrids in the quantum setting. This means $B$ breaks the security of $G$ with only exponentially small probability. All existing security proofs for pseudorandom functions from standard assumptions suffer from similar weaknesses. 


\subsection{Our Results}

We answer the question of how to construct a function that looks random to quantum adversaries. The answer is simple: many of the constructions of standard-secure pseudorandom functions are in fact quantum-secure. However, as explained above, new techniques are required to actually prove security.

We start by showing that, given the existence of a standard-secure pseudorandom function, there are standard-secure pseudorandom functions that are not quantum-secure. Thus a standard-secure PRF may not be secure as a QPRF.

Next, for several classical constructions of pseudorandom functions, we now can show how to modify the classical security proof to prove quantum security. Our general technique is as follows: first define a seemingly stronger security notion for the underlying cryptographic primitive. Next, use ideas from the classical proof to show that any adversary $A$ that breaks the quantum-security of the pseudorandom function can be turned into an adversary $B$ that breaks this stronger notion of security for the primitive. Lastly, use new techniques to show the equivalence of this stronger notion of security and the standard notion of security in the quantum setting.

We use this approach to prove the security of the following pseudorandom functions:

- The construction from length-doubling pseudorandom generators (PRGs) due to Goldreich, Goldwasser, and Micali [GGM86]. Since pseudorandom generators can be built from one-way functions in the quantum setting, this shows that one-way functions secure against quantum adversaries imply quantum-secure pseudorandom functions.

- The construction from pseudorandom synthesizers due to Naor and Reingold [NR95].

- The direct construction based on the Learning With Errors problem due to Banerjee, Peikert, and Rosen [BPR11].

\subsection{Are Quantum Oracles Better Than Samples?}

In the GGM proof, the first hybrid argument over the levels of the tree essentially transforms an adversary for PRF into an algorithm solving the following problem: distinguish a random oracle from an oracle whose outputs are random values from the underlying pseudorandom generator. The next hybrid argument shows how to use such an algorithm do distinguish a single random value from a single output of the pseudorandom generator, thus breaking the security of the pseudorandom generator.

The first hybrid argument carries over into the quantum setting, but it is this second hybrid that causes difficulties for the reasons outlined above. To complete the security proof, we need to show that having quantum access to an oracle whose outputs are drawn from a distribution is no better than having access to a single sample from the same distribution. We show exactly that:

Theorem 1.1. Let $D_{1}$ and $D_{2}$ be efficiently sampleable distributions on a set $\mathcal{Y}$, and let $\mathcal{X}$ be some other set. Let $O_{1}$ and $O_{2}$ be the distributions of functions from $\mathcal{X}$ to $\mathcal{Y}$ where for each $x \in \mathcal{X}, O_{i}(x)$ is chosen independently according to $D_{i}$. Then if $A$ is an efficient quantum algorithm that uses quantum queries to distinguish oracles drawn from $\mathrm{O}_{1}$ from oracles drawn from $\mathrm{O}_{2}$, we can construct an efficient quantum algorithm $B$ that distinguishes samples from $D_{1}$ and $D_{2}$. 
In the classical case, any algorithm $A$ making $q$ queries to an oracle $O$ only sees $q$ outputs. Thus, given $q$ samples from $D_{1}$ or $D_{2}$, we can lazily simulate the oracles $O_{1}$ or $O_{2}$, getting an algorithm that distinguishes $q$ samples of $D_{1}$ from $q$ samples of $D_{2}$. A simple hybrid argument shows how to get an algorithm that distinguishes one sample.

In the quantum setting, any quantum algorithm $A$ making even a single quantum query to $O_{i}$ gets to "see" all the outputs at once, meaning we need exponentially many samples to simulate $O_{i}$ exactly. However, while we cannot lazily simulate the oracles $O_{i}$ given $q$ samples from $D_{i}$, we can approximately simulate $O_{i}$ given polynomially many samples from $D_{i}$. Basically, for each input, set the output to be one of the samples, chosen at random from the collection of samples. While not quite the oracle $O_{i}$, we show that this is sufficiently indistinguishable from $O_{i}$. Thus, we can use $A$ to distinguish a polynomial number of samples of $D_{1}$ from the same number of samples of $D_{2}$. Like in the classical case, a simple hybrid argument shows how to distinguish just one sample.

\section{Preliminaries and Notation}

We say that $\epsilon=\epsilon(n)$ is negligible if, for all polynomials $p(n), \epsilon(n)<1 / p(n)$ for large enough $n$.

For an integer $k$, we will use non-standard notation and write $[k]=\{0, \ldots, k-1\}$ to be the set of non-negative integers less than $k$. We write the set of all $n$ bit strings as $[2]^{n}$. Let $x=x_{1} \ldots x_{n}$ be a string of length $n$. We write $x_{[a, b]}$ to denote the substring $x_{a} x_{a+1} \ldots x_{b}$.

\subsection{Functions and Probabilities}

Given two sets $\mathcal{X}$ and $\mathcal{Y}$, define $\mathcal{Y}^{\mathcal{X}}$ as the set of functions $f: \mathcal{X} \rightarrow \mathcal{Y}$. If a function $f$ has maps $\mathcal{X}$ to $\mathcal{Y} \times \mathcal{Z}$, we can think of $f$ as two functions: one that maps $\mathcal{X}$ to $\mathcal{Y}$ and one that maps $\mathcal{X}$ to $\mathcal{Z}$. In other words, we can equate the sets of functions $(\mathcal{Y} \times \mathcal{Z})^{\mathcal{X}}$ and $\mathcal{Y}^{\mathcal{X}} \times \mathcal{Z}^{\mathcal{X}}$.

Given $f \in \mathcal{Y}^{\mathcal{X}}$ and $g \in \mathcal{Z}^{\mathcal{Y}}$, let $g \circ f$ be the composition of $f$ and $g$. That is, $g \circ f(x)=g(f(x))$. If $\mathcal{F} \subseteq \mathcal{Y}^{\mathcal{X}}$, let $g \circ \mathcal{F}$ be the set of functions $g \circ f$ for $f \in \mathcal{F}$. Similarly, if $\mathcal{G} \subseteq \mathcal{Z}^{\mathcal{Y}}, \mathcal{G} \circ f$ is the set of functions $f \circ g$ where $g \in \mathcal{G}$. Define $\mathcal{G} \circ \mathcal{F}$ accordingly.

Given a distribution $D$ and some event event, we write $\operatorname{Pr}_{x \leftarrow D}$ [event] to represent the probability that event happens when $x$ is drawn from $D$. For a given set $\mathcal{X}$, we will sometimes abuse notation and write $\mathcal{X}$ to denote the uniform distribution on $\mathcal{X}$.

Given a distribution $D$ on $\mathcal{Y}^{\mathcal{X}}$ and a function $g \in \mathcal{Z}^{\mathcal{Y}}$, define the distribution $g \circ D$ over $\mathcal{Z}^{\mathcal{X}}$ where we first draw $f$ from $D$, and output the composition $g \circ f$. Given $f \in \mathcal{Y}^{\mathcal{X}}$ and a distribution $E$ over $\mathcal{Z}^{\mathcal{X}}$, define $E \circ f$ and $E \circ D$ accordingly.

Given a distribution $D$ on a set $\mathcal{Y}$, and another set $\mathcal{X}$, define $D^{\mathcal{X}}$ as the distribution on $\mathcal{Y}^{\mathcal{X}}$ where the output for each input is chosen independently according to $D$.

The distance between two distributions $D_{1}$ and $D_{2}$ over a set $\mathcal{X}$ is

$$
\left|D_{1}-D_{2}\right|=\sum_{x \in \mathcal{X}}\left|D_{1}(x)-D_{2}(x)\right| .
$$

If $\left|D_{1}-D_{2}\right| \leq \epsilon$, we say $D_{1}$ and $D_{2}$ are $\epsilon$-close. If $\left|D_{1}-D_{2}\right| \geq \epsilon$, we say they are $\epsilon$-far. 


\subsection{Quantum Computation}

Here we state some basic facts about quantum computation needed for the paper, and refer the reader to Nielsen and Chuang [NC00] for a more in depth discussion.

Fact 1. Any classical efficiently computable function $f$ can be implemented efficiently by a quantum computer. Moreover, $f$ can be implemented as an oracle which can be queried on quantum superpositions.

The following is a result from Zhandry [Zha12]:

Fact 2. For any sets $\mathcal{X}$ and $\mathcal{Y}$, we can efficiently "construct" a random oracle from $\mathcal{X}$ to $\mathcal{Y}$ capable of handling q quantum queries, where $q$ is a polynomial. More specifically, the behavior of any quantum algorithm making at most q queries to a 2q-wise independent function is identical to its behavior when the queries are made to a random function.

Given an efficiently sampleable distribution $D$ over a set $\mathcal{Y}$, we can also "construct" a random function drawn from $D^{\mathcal{X}}$ as follows: Let $\mathcal{Z}$ be the set of randomness used to sample from $D$, and let $f(r)$ be the element $y \in \mathcal{Y}$ obtained using randomness $r \in \mathcal{Z}$. Then $D^{\mathcal{X}}=f \circ \mathcal{Z}^{\mathcal{X}}$, so we first construct a random function $O^{\prime} \in \mathcal{Z}^{\mathcal{X}}$, and let $O(x)=f\left(O^{\prime}(x)\right)$.

We will denote a quantum algorithm $A$ given classical oracle access to an oracle $O$ as $A^{O}$. If $A$ has quantum access, we will denote this as $A^{|O\rangle}$.

\subsection{Cryptographic Primitives}

In this paper, we always assume the adversary is a quantum computer. However, for any particular primitive, there may be multiple definitions of security, based on how the adversary is allowed to interact with the primitive. Here we define pseudorandom functions and two security notions, as well as two definitions of indistinguishability for distributions. The definitions of pseudorandom generators and synthesizers appear in the relevant sections.

Definition 2.1 (PRF). A pseudorandom function is a function PRF : $\mathcal{K} \times \mathcal{X} \rightarrow \mathcal{Y}$, where $\mathcal{K}$ is the key-space, and $\mathcal{X}$ and $\mathcal{Y}$ are the domain and range. $\mathcal{K}, \mathcal{X}$, and $\mathcal{Y}$ are implicitly functions of the security parameter $n$. We write $y=\operatorname{PRF}_{k}(x)$.

Definition 2.2 (Standard-Security). A pseudorandom function PRF is standard-secure if no efficient quantum adversary $A$ making classical queries can distinguish between a truly random function and the function $\mathrm{PRF}_{k}$ for a random $k$. That is, for every such $A$, there exists a negligible function $\epsilon=\epsilon(n)$ such that

$$
\left|\operatorname{Pr}_{k \leftarrow \mathcal{K}}\left[A^{\mathrm{PRF}_{k}}()=1\right]-\operatorname{Pr}_{O \leftarrow \mathcal{Y}}\left[A^{O}()=1\right]\right|<\epsilon .
$$

Definition 2.3 (Quantum-Security). A pseudorandom function PRF is quantum-secure if no efficient quantum adversary A making quantum queries can distinguish between a truly random function and the function $\mathrm{PRF}_{k}$ for a random $k$.

We call such quantum-secure pseudorandom functions Quantum Random Functions, or QPRFs.

We now provide some definitions of indistinguishablility for distributions. The standard notion of indistinguishability is that no efficient quantum algorithm can distinguish a sample of one distribution from a sample of the other: 
Definition 2.4 (Indistinguishability). Two distributions $D_{1}$ and $D_{2}$ over a set $\mathcal{Y}$ are computationally (resp. statistically) indistinguishable if no efficient (resp. computationally unbounded) quantum algorithm $A$ can distinguish a sample of $D_{1}$ from a sample of $D_{2}$. That is, for all such $A$, there is a negligible function $\epsilon$ such that

$$
\left|\operatorname{Pr}_{y \leftarrow D_{1}}[A(y)=1]-\operatorname{Pr}_{y \leftarrow D_{2}}[A(y)=1]\right|<\epsilon .
$$

For our work, we will also need a new, seemingly stronger notion of security, which we call oracle-indistinguishability. The idea is that no efficient algorithm can distinguish between oracles whose outputs are distributed according to either $D_{1}$ or $D_{2}$ :

Definition 2.5 (Oracle-Indistinguishability). Two distributions $D_{1}$ and $D_{2}$ over a set $\mathcal{Y}$ are computationally (resp. statistically) oracle-indistinguishable if, for all sets $\mathcal{X}$, no efficient (resp. computationally unbounded) quantum algorithm $B$ can distinguish $D_{1}^{\mathcal{X}}$ from $D_{2}^{\mathcal{X}}$ using a polynomial number of quantum queries. That is, for all such $B$ and $\mathcal{X}$, there is a negligible function $\epsilon$ such that

$$
\left|\operatorname{Pr}_{O \leftarrow D_{1}^{\mathcal{X}}}\left[B^{|O\rangle}()=1\right]-\operatorname{Pr}_{O \leftarrow D_{2}^{\mathcal{X}}}\left[B^{|O\rangle}()=1\right]\right|<\epsilon .
$$

For this paper, we will primarily be discussing computationally bounded adversaries, so we will normally take indistinguishability and oracle-indistinguishability to mean the computational versions.

These these definitions of indistinguishability in hand, we can now formulate Theorem 1.1 as follows:

Theorem 1.1. Let $D_{1}$ and $D_{2}$ be efficiently sampleable distributions over a set $\mathcal{Y}$. Then $D_{1}$ and $D_{2}$ are indistinguishable if and only if they are also oracle-indistinguishable.

\section{Separation Result}

In this section, we show our separation result:

Theorem 3.1. If secure PRFs exist, then there are standard-secure PRFs that are not QPRFs.

Proof. Let PRF be a standard-secure pseudorandom function with key-space $\mathcal{K}$, domain $\mathcal{X}$, and co-domain $\mathcal{Y}$. We will construct a new pseudorandom function that is periodic with some large, secret period. Classical adversaries will not be able to detect the period, and thus cannot distinguish this new function from random. However, an adversary making quantum queries can detect the period, and thus distinguish our new function from random.

Interpret $\mathcal{X}$ as $[N]$, where $N$ is the number of elements in $\mathcal{X}$. Assume without loss of generality that $\mathcal{Y}$ contains at least $N^{2}$ elements (if not, we can construct a new pseudorandom function with smaller domain but larger range in a standard way). We now construct a new pseudorandom function $\mathrm{PRF}_{(k, a)}^{\prime}(x)=\mathrm{PRF}_{k}(x \bmod a)$ where:

- The key space of $\mathrm{PRF}^{\prime}$ is $\mathcal{K}^{\prime}=\mathcal{K} \times \mathcal{A}$ where $\mathcal{A}$ is the set of primes between $N / 2$ and $N$. That is, a key for $\mathrm{PRF}^{\prime}$ is a pair $(k, a)$ where $k$ is a key for PRF, and $a$ is a prime in the range $(N / 2, N]$. 
- The domain is $\mathcal{X}^{\prime}=\left[N^{\prime}\right]$ where $N^{\prime}$ is the smallest power of 2 greater than $4 N^{2}$.

The following two claims are proved in Appendix A:

Claim 1. If PRF is standard-secure, then so is $\mathrm{PRF}^{\prime}$.

Sketch of Proof. Since PRF is a standard-secure pseudorandom function, we can replace it with a truly random function in the definition of $\mathrm{PRF}^{\prime}$, and no efficient adversary making classical queries will notice. But we are then left with a function that has a large random period where every value in the period is chosen randomly. This function will look truly random unless the adversary happens to query two points that differ by a multiple of the period. But by the birthday bound, this will only happen with negligible probability.

Claim 2. If $\mathrm{PRF}$ is quantum-secure, then $\mathrm{PRF}^{\prime}$ is not.

Sketch of Proof. If we allow quantum queries to $\mathrm{PRF}^{\prime}$, we can use the period finding algorithm of Boneh and Lipton [BL95] to find $a$. With $a$, it is easy to distinguish PRF $^{\prime}$ from a random oracle. Unfortunately, the period finding algorithm requires $\mathrm{PRF}^{\prime}$ to have some nice properties, but these properties are satisfied if PRF is quantum-secure.

Thus one of PRF and $\mathrm{PRF}^{\prime}$ is standard-secure but not quantum-secure, as desired.

We have shown that for pseudorandom functions, security against classical queries does not imply security against quantum queries. In the next sections, we will show, however, that several of the standard constructions in the literature are nevertheless quantum-secure.

\section{Pseudorandom Functions from Pseudorandom Generators}

We give the construction of pseudorandom functions from pseudorandom generators due to Goldreich, Goldwasser, and Micali [GGM86], the so-called GGM construction. We also prove its security in a new way that makes sense in the quantum setting. First, we define pseudorandom generators:

Definition 4.1 (PRG). A pseudorandom generator $(P R G)$ is a function $G: \mathcal{X} \rightarrow \mathcal{Y}$. $\mathcal{X}$ and $\mathcal{Y}$ are implicitly indexed by the security parameter $n$.

Definition 4.2 (Standard-Security). A pseudorandom generator $G$ is standard-secure if the distributions $G \circ \mathcal{X}$ and $\mathcal{Y}$ are computationally indistinguishable.

Construction 1 (GGM-PRF). Let $G: \mathcal{K} \rightarrow \mathcal{K}^{2}$ be a length-doubling pseudorandom generator. Write $G(x)=\left(G_{0}(x), G_{1}(x)\right)$ where $G_{0}, G_{1}$ are functions from $\mathcal{K}$ to $\mathcal{K}$. Then we define the $G G M$ pseudorandom function $\mathrm{PRF}: \mathcal{K} \times[2]^{n} \rightarrow \mathcal{K}$ where

$$
\operatorname{PRF}_{k}(x)=G_{x_{1}}\left(\ldots G_{x_{n-1}}\left(G_{x_{n}}(k)\right) \ldots\right) .
$$

That is, the function PRF takes a key $k$ in $\mathcal{K}$ and an $n$-bit input string. It first applies $G$ to $k$. It keeps the left or right half of the output depending on whether the last bit of the input is 0 or 1. What remains is an element in $\mathcal{K}$, so the function applies $G$ again, keeps the left or right half depending on the second-to-last bit, and so on.

As described in the introduction, the standard proof of security fails to prove quantum-security. Using Theorem 1.1, we show how to work around this problem. We defer the proof of Theorem 1.1 
to Section 7, and instead assume it is true. We first define a stronger notion of security for pseudorandom generators, which we call oracle-security:

Definition 4.3 (Oracle-Security). A pseudorandom generator $G: \mathcal{X} \rightarrow \mathcal{Y}$ is oracle-secure if the distributions $G \circ \mathcal{X}$ and $\mathcal{Y}$ are oracle-indistinguishable.

$G \circ \mathcal{X}$ is efficiently sampleable since we can sample a random value in $\mathcal{X}$ and apply $G$ to it. Then, $G \circ \mathcal{X}$ and $\mathcal{Y}$ are both efficiently sampleable, so Theorem 1.1 gives:

Corollary 4.4. If $G$ is a secure PRG, then it is also oracle-secure.

We now can prove the security of Construction 1 .

Theorem 4.5. If $G$ is a standard-secure PRG, then PRF from Construction 1 is a QPRF.

Proof. We adapt the security proof of Goldreich et al. to convert any adversary for PRF into an adversary for the oracle-security of $G$. Then Corollary 4.4 shows that this adversary is impossible under the assumption that $G$ is standard-secure.

Suppose a quantum adversary $A$ distinguishes PRF from a random oracle with probability $\epsilon$. Define hybrids $H_{i}$ as follows: Pick a random function $P \leftarrow \mathcal{K}^{[2]^{n-i}}$ (that is, random function from $(n-i)$-bit strings into $\mathcal{K})$ and give $A$ the oracle

$$
O_{i}(x)=G_{x_{1}}\left(\ldots G_{x_{i}}\left(P\left(x_{[i+1, n]}\right)\right) \ldots\right) .
$$

$H_{0}$ is the case where $A$ 's oracle is random. When $i=n, P \leftarrow \mathcal{K}^{[2]^{n-i}}$ is a random function from the set containing only the empty string to $\mathcal{K}$, and hence is associated with the image of the empty string, a random element in $\mathcal{K}$. Thus $H_{n}$ is the case where $A$ 's oracle is PRF. Let $\epsilon_{i}$ be the probability $A$ distinguishes $H_{i}$ from $H_{i+1}$. That is,

$$
\epsilon_{i}=\operatorname{Pr}\left[A^{\left|O_{i}\right\rangle}()=1\right]-\operatorname{Pr}\left[A^{\left|O_{i+1}\right\rangle}()=1\right] .
$$

A simple hybrid argument shows that $\left|\sum_{i} \epsilon_{i}\right|=\epsilon$.

We now construct a quantum algorithm $B$ breaking the oracle-security of $G$. $B$ is given quantum access to an oracle $P:[2]^{n-1} \rightarrow \mathcal{K}^{2}$, and distinguishes $P \leftarrow\left(\mathcal{K}^{2}\right)^{[2]^{n-1}}$ from $P \leftarrow G \circ \mathcal{K}^{[2]^{n-1}}$. That is, $B$ is given either a random function from $(n-1)$-bit strings into $\mathcal{K}^{2}$, or $G$ applied to a random function from $(n-1)$-bit strings into $\mathcal{K}$, and distinguishes the two cases as follows:

- Pick a random $i$ in $\{0, \ldots, n-1\}$

- Let $P^{(i)}:[2]^{n-i-1} \rightarrow \mathcal{K}^{2}$ be the oracle $P^{(i)}(x)=P\left(0^{i} x\right)$

- Write $P^{(i)}$ as $\left(P_{0}^{(i)}, P_{1}^{(i)}\right)$ where $P_{b}^{(i)}:[2]^{n-i-1} \rightarrow \mathcal{K}$ are the left and right halfs of the output of $P^{(i)}$.

- Construct the oracle $O:[2]^{n} \rightarrow \mathcal{K}$ where

$$
O(x)=G_{x_{1}}\left(\ldots G_{x_{i}}\left(P_{x_{i+1}}^{(i)}\left(x_{[i+2, n]}\right)\right) \ldots\right) .
$$

- Simulate $A$ with oracle $O$, and output whatever $A$ outputs. 
Notice that each quantum query to $O$ results in one quantum query to $P$, so $B$ makes the same number of queries that $A$ does.

Fix $i$, and let $B_{i}$ be the algorithm $B$ using this $i$. In the case where $P$ is truly random, so is $P^{(i)}$, as are $P_{0}^{(i)}$ and $P_{1}^{(i)}$. Thus $O=O_{i}$, the oracle in hybrid $H_{i}$. When $P$ is drawn from $G \circ \mathcal{K}^{[2]^{n-1}}$, then $P^{(i)}$ is distributed according to $G \circ \mathcal{K}^{[2]^{n-i-1}}$, and so $P_{b} \leftarrow G_{b} \circ \mathcal{K}^{[2]^{n-i-1}}$. Thus $O=O_{i+1}$, the oracle in hybrid $H_{i+1}$. For fixed $i$, we then have that the quantity

$$
\operatorname{Pr}_{P \leftarrow\left(\mathcal{K}^{2}\right)^{[2]^{n-1}}}\left[B_{i}^{|P\rangle}()=1\right]-\operatorname{Pr}_{P \leftarrow G \circ \mathcal{K}^{[2]^{n-1}}}\left[B_{i}^{|P\rangle}()=1\right]
$$

is equal to $\epsilon_{i}$. Averaging over all $i$ and taking the absolute value, we have that the distinguishing probability of $B$,

$$
\left|\operatorname{Pr}_{P \leftarrow\left(\mathcal{K}^{2}\right)^{[2]^{n-1}}}\left[B^{|P\rangle}()=1\right]-\operatorname{Pr}_{P \leftarrow G \circ \mathcal{K}^{[2]^{n-1}}}\left[B^{|P\rangle}()=1\right]\right|,
$$

is equal to

$$
\left|\frac{1}{n} \sum_{i} \epsilon_{i}\right|=\epsilon / n
$$

Thus $B$ breaks the oracle security of $G$ with probability only polynomially smaller than the probability $A$ distinguishes PRF from a random oracle.

\section{Pseudorandom Functions from Synthesizers}

In this section, we show that the construction of pseudorandom functions from pseudorandom synthesizers due to Naor and Reingold [NR95] is quantum-secure.

Definition 5.1 (Synthesizer). A pseudorandom synthesizer is a function $S: \mathcal{X}^{2} \rightarrow \mathcal{Y}$. X and $\mathcal{Y}$ are implicitly indexed by the security parameter $n$.

Definition 5.2 (Standard-Security). A pseudoreandom synthesizer $S: \mathcal{X}^{2} \rightarrow \mathcal{Y}$ is standard-secure if, for any set $\mathcal{Z}$, no efficient quantum algorithm $A$ making classical queries can distinguish a random function from $O\left(z_{1}, z_{2}\right)=S\left(O_{1}\left(z_{1}\right), O_{2}\left(z_{2}\right)\right)$ where $O_{b} \leftarrow \mathcal{X}^{\mathcal{Z}}$. That is, for any such $A$ and $\mathcal{Z}$, there exists a negligible function $\epsilon$ such that

$$
\left|\operatorname{Pr}_{\substack{O_{1} \leftarrow \mathcal{X} \mathcal{Z} \\ O_{2} \leftarrow \mathcal{X}^{\mathcal{Z}}}}\left[A^{S\left(O_{1}, O_{2}\right)}()=1\right]-\operatorname{Pr}_{O \leftarrow \mathcal{Y}^{\mathcal{Z} \times \mathcal{Z}}}\left[A^{O}()=1\right]\right|<\epsilon,
$$

where $S\left(O_{1}, O_{2}\right)$ means the oracle that maps $\left(z_{1}, z_{2}\right)$ into $S\left(O_{1}\left(z_{1}\right), O_{2}\left(z_{2}\right)\right)$.

Construction 2 (NR-PRF). Given a pseudorandom synthesizer $S: \mathcal{X}^{2} \rightarrow \mathcal{X}$, let $\ell$ be an integer and $n=2^{\ell}$. We let $\operatorname{PRF}_{k}(x)=\operatorname{PRF}_{k}^{(\ell)}(x)$ where $\operatorname{PRF}^{(i)}:\left(\mathcal{X}^{2 \times 2^{i}}\right) \times[2]^{2^{i}} \rightarrow \mathcal{X}$ is defined as

$$
\begin{aligned}
\operatorname{PRF}_{a_{1,0}, a_{1,1}}^{(0)}(x) & =a_{1, x} \\
\mathrm{PRF}_{A_{1}^{(i-1)}, A_{2}^{(i-1)}}^{(i)}(x) & =S\left(\mathrm{PRF}_{A_{1}^{(i-1)}}^{(i-1)}\left(x_{\left[1,2^{i-1}\right]}\right),\right. \\
& \left.\operatorname{PRF}_{A_{2}^{(i-1)}}^{(i-1)}\left(x_{\left[2^{i-1}+1,2^{i}\right]}\right)\right),
\end{aligned}
$$


where

$$
\begin{aligned}
& A_{1}^{(i-1)}=\left(a_{1,0}, a_{1,1}, a_{2,0}, a_{2,1}, \ldots, a_{2^{i-1}, 0}, a_{2^{i-1}, 1}\right) \\
& A_{2}^{(i-1)}=\left(a_{2^{i-1}+1,0}, a_{2^{i-1}+1,1}, a_{2,0}, a_{2,1}, \ldots, a_{2^{i}, 0}, a_{2^{i}, 1}\right)
\end{aligned}
$$

That is, PRF takes a key $k$ consisting of $2 \times 2^{\ell}$ elements of $\mathcal{X}$, and takes bit strings $x$ of length $2^{\ell}$ as input. It uses $x$ to select $2^{\ell}$ of the elements in the key, and pairs them off. It then applies $S$ to each of the pairs, obtaining $2^{\ell-1}$ elements of $\mathcal{X}$. Next, PRF pairs these elements and applies $S$ to these pairs again, and continues in this way until there is one element left, which becomes the output.

The following theorem is proved in Appendix E:

Theorem 5.3. If $S$ is a standard-secure synthesizer, then PRF from Construction 2 is a $Q P R F$.

Sketch of Proof. The proof is very similar to that of the security of the GGM construction: we define a new notion of security for synthesizers, called quantum-security, and use the techniques of Naor and Reingold to prove that quantum-security implies that Construction 2 is quantum secure. Unlike the GGM case, the equivalence of quantum- and standard-security for synthesizers is not an immediate consequence of Theorem 1.1. Nevertheless, we prove the equivalence, completing the proof of security for Construction 2 .

\section{Direct Construction of Pseudorandom Functions}

In this section, we present the construction of pseudorandom functions from Banerjee, Peikert, and Rosen [BPR11]. We show that this construction is quantum-secure.

Let $p, q$ be integers with $q>p$. Let $\lfloor x\rceil_{p}$ be the map from $\mathbb{Z}_{q}$ into $\mathbb{Z}_{p}$ defined by first rounding $x$ to the nearest multiple of $q / p$, and then interpreting the result as an element of $\mathbb{Z}_{p}$. More precisely, $\lfloor x\rceil_{p}=\lfloor(p / q) x\rceil \bmod p$ where the multiplication and division in $(p / q) x$ are computed in $\mathbb{R}$.

Construction 3. Let $p, q, m, \ell$ be integers with $q>p$. Let $\mathcal{K}=\mathbb{Z}_{q}^{n \times m} \times\left(\mathbb{Z}^{n \times n}\right)^{\ell}$. We define $\mathrm{PRF}: \mathcal{K} \times[2]^{\ell} \rightarrow \mathbb{Z}_{p}^{m \times n}$ as follows: For a key $k=\left(\mathbf{A},\left\{\mathbf{S}_{i}\right\}\right)$, let

$$
\operatorname{PRF}_{k}(x)=\left\lfloor\mathbf{A}^{t} \prod_{i=1}^{\ell} \mathbf{S}_{i}^{x_{i}}\right\rceil_{p} .
$$

The function PRF uses for a key an $n \times m$ matrix $\mathbf{A}$ and $\ell$ different $n \times n$ matrices $\mathbf{S}_{i}$, where elements are integers $\bmod q$. It uses its $\ell$-bit input to select a subset of the $\mathbf{S}_{i}$, which it multiplies together. The product is then multiplied by the transpose of $\mathbf{A}$, and the whose result is rounded $\bmod p$.

Next is an informal statement of the security of PRF, whose proof appears in Appendix F:

Theorem 6.1. Let PRF be as in Construction 3. For an appropriate choice of integers $p, q, m, \ell$ and distribution $\chi$ on $\mathbb{Z}$, if we draw $\mathbf{A} \leftarrow \mathbb{Z}_{q}^{n \times m}$ and $S_{i} \leftarrow \chi^{n \times n}$ and the Learning With Errors (LWE) problem is hard for modulus $q$ and distribution $\chi$, then PRF is a QPRF. 
Sketch of Proof. We follow the ideas from the previous sections and define a new notion of hardness for LWE, which we call oracle-hard, and show its equivalence to standard hardness. We then show that oracle-hardness implies Construction 3 is quantum-secure. This part is similar to the proof of Banerjee et al., with some changes to get it to work in the quantum setting.

\section{Distinguishing Oracle Distributions}

In this section, we describe some tools for arguing that a quantum algorithm cannot distinguish between two oracle distributions, culminating in a proof for Theorem 1.1. Let $\mathcal{X}$ and $\mathcal{Y}$ be sets. We start by recalling two theorems of Zhandry [Zha12]:

Theorem 7.1. Let $A$ be a quantum algorithm making $q$ quantum queries to an oracle $H: \mathcal{Y}^{\mathcal{X}}$. If we draw $H$ from some distribution $D$, then for every $z$, the quantity $\operatorname{Pr}_{H \leftarrow D}\left[A^{|H\rangle}()=z\right]$ is a linear combination of the quantities $\operatorname{Pr}_{H \leftarrow D}\left[H\left(x_{i}\right)=r_{i} \forall i \in\{1, \ldots, 2 q\}\right]$ for all possible settings of the $x_{i}$ and $r_{i}$.

Theorem 7.2. Fix $q$, and let $D_{\lambda}$ be a family of distributions on $\mathcal{Y}^{\mathcal{X}}$ indexed by $\lambda \in[0,1]$. Suppose there is an integer $d$ such that for every $2 q$ pairs $\left(x_{i}, r_{i}\right) \in \mathcal{X} \times \mathcal{Y}$, the function $p(\lambda)=$ $\operatorname{Pr}_{H \leftarrow D_{\lambda}}\left[H\left(x_{i}\right)=r_{i} \forall i \in\{1, \ldots, 2 q\}\right]$ is a polynomial of degree at most $d$ in $\lambda$. Then for any quantum algorithm $A$ making q quantum queries, the output distributions under $D_{\lambda}$ and $D_{0}$ are $2 \lambda d^{2}$-close.

We now show a similar result:

Theorem 7.3. Fix $q$, and let $E_{r}$ be a family of distributions on $\mathcal{Y}^{\mathcal{X}}$ indexed by $r \in \mathbb{Z}^{+} \bigcup\{\infty\}$. Suppose there is an integer $d$ such that for every $2 q$ pairs $\left(x_{i}, r_{i}\right) \in \mathcal{X} \times \mathcal{Y}$, the function $p(\lambda)=$ $\operatorname{Pr}_{H \leftarrow E_{1 / \lambda}}\left[H\left(x_{i}\right)=r_{i} \forall i \in\{1, \ldots, 2 q\}\right]$ is a polynomial of degree at most $d$ in $\lambda$. Then for any quantum algorithm $A$ making q quantum queries, the output distributions under $E_{r}$ and $E_{\infty}$ are $\pi^{2} d^{3} / 3 r$-close.

Sketch of Proof. Let $D_{\lambda}=E_{1 / \lambda}$. We see that the conditions of Theorems 7.3 and 7.2 are identical, with the following exception: Theorem 7.2 requires $D_{\lambda}$ to be a distribution for all $\lambda \in[0,1]$, while Theorem 7.3 only requires $D_{\lambda}$ to be a distribution when $1 / \lambda$ is an integer (and when $\lambda=0$ ). The proof is thus similar in flavor to that of Theorem 7.2, with the following exception: the proof of Theorem 7.2 uses well-known bounds on polynomials $f$ where $f(x) \in[0,1]$ for all $x \in[0,1]$. However, we need similar bounds on polynomials $f$ where $f(x)$ is only required to be in $[0,1]$ for $x$ where $1 / x$ is an integer. Such polynomials are far less understood, and we need to prove suitable bounds under these relaxed assumptions. The proof is in Appendix B.

In the next section, we apply Theorem 7.3 to a new class of distributions.

\subsection{Small-Range Distributions}

We now apply Theorem 7.3 to a new distribution on oracles, which we call small-range distributions. Given a distribution $D$ on $\mathcal{Y}$, define $\operatorname{SR}_{r}^{D}(\mathcal{X})$ as the following distribution on functions from $\mathcal{X}$ to $\mathcal{Y}$ :

- For each $i \in[r]$, chose a random value $y_{i} \in \mathcal{Y}$ according to the distribution $D$.

- For each $x \in \mathcal{X}$, pick a random $i \in[r]$ and set $O(x)=y_{i}$. 
We will often omit the domain $\mathcal{X}$ when is is clear from context.

The following is proved in Appendix C:

Lemma 7.4. Fix $k$. For any $\mathcal{X}$, the probabilities in each of the marginal distributions of $\operatorname{SR}_{r}^{D}(\mathcal{X})$ over $k$ inputs are polynomials in $1 / r$ of degree $k$.

An alternate view of this function is to choose $g \leftarrow D^{[r]}$ and $f \leftarrow[r]^{\mathcal{X}}$, and output the composition $g \circ f$. That is, $\mathrm{SR}_{r}^{D}(\mathcal{X})=D^{[r]} \circ[r]^{\mathcal{X}}$. In other words, we choose a random function $f$ from $\mathcal{X}$ to $[r]$, and compose it with another random function $g$ from $[r]$ to $\mathcal{Y}$, where outputs are distributed according to $D$. We call this distribution a small-range distribution because the set of images of any function drawn from the distribution is bounded to at most $r$ points, which for $r<<\mathcal{Y}$ will be a small subset of the co-domain. Notice that, as $r$ goes to infinity, $f$ will be injective with probability 1 , and hence for each $x, g(f(x))$ will be distributed independently according to $D$. That is, $\mathrm{SR}_{\infty}^{D}(\mathcal{X})=D^{\mathcal{X}}$. We can then use Theorem 7.3 to bound the ability of any quantum algorithm to distinguish $\mathrm{SR}_{r}^{D}(\mathcal{X})$ from $\mathrm{SR}_{\infty}^{D}(\mathcal{X})=D^{\mathcal{X}}$ :

Corollary 7.5. The output distributions of a quantum algorithm making q quantum queries to an oracle either drawn from $\operatorname{SR}_{r}^{D}(\mathcal{X})$ or $D^{\mathcal{X}}$ are $\ell(q) / r$-close, where $\ell(q)=\pi^{2}(2 q)^{3} / 3<27 q^{3}$.

We observe that this bound is tight: in Appendix D we show that the quantum collision finding algorithm of Brassard, Høyer, and Tapp [BHT97] can be used to distinguish $\mathrm{SR}_{r}^{D}(\mathcal{X})$ from $D^{\mathcal{X}}$ with optimal probability. This shows that Theorem 7.3 is tight.

\subsection{The Equivalence of Indistinsguishability and Oracle-Indistinguishability}

We now use the above techniques to explore the relationship between indistinguishability and oracle-indistinguishability and to prove Theorem 1.1. Clearly, oracle-indistinguishability implies standard indistinguishability: if $A$ distinguishes $D_{1}$ from $D_{2}$, then the algorithm $B^{|O\rangle}()$ that picks any $x \in \mathcal{X}$ and returns $A(O(x))$ breaks the oracle-indistinguishability.

In the other direction, in the classical world, if $B$ makes $q$ queries to $O$, we can simulate $O$ using $q$ samples, and do a hybrid across the samples. This results in an algorithm that breaks the standard indistinuishability. However, in the quantum world, each query might be over a superposition of exponentially many inputs. Therefore there will be exponentially many hybrids, causing the proof to fail.

In the statistical setting, this question has been answered by Boneh et al. [BDF $\left.{ }^{+} 11\right]$. They show that if a (potentially unbounded) quantum adversary making $q$ queries distinguishes $D_{1}^{\mathcal{X}}$ from $D_{2}^{\mathcal{X}}$ with probability $\epsilon$, then $D_{1}$ and $D_{2}$ must $\Omega\left(\epsilon^{2} / q^{4}\right)$-far. We now have the tools to extend this result to the computational setting (and improve the result for the statistical setting in the process) by proving Theorem 1.1.

Proof of Theorem 1.1. Let $B$ be an (efficient) quantum adversary that distinguishes $D_{1}^{\mathcal{X}}$ from $D_{2}^{\mathcal{X}}$ with non-negligible probability $\epsilon$, for distributions $D_{1}$ and $D_{2}$ over $\mathcal{Y}$. That is, there is some set $\mathcal{X}$ such that

$$
\left|\operatorname{Pr}_{O \leftarrow D_{1}^{\mathcal{X}}}\left[B^{|O\rangle}()=1\right]-\operatorname{Pr}_{O \leftarrow D_{2}^{\mathcal{X}}}\left[B^{|O\rangle}()=1\right]\right|=\epsilon .
$$

Our goal is to construct an (efficient) quantum algorithm $A$ that distinguishes a sample of $D_{1}$ from a sample of $D_{2}$. To this end, choose $r$ so that $\ell(q) / r=\epsilon / 4$, where $\ell(q)$ is the polynomial from 
Corollary 7.5. That is, $r=4 \ell(q) / \epsilon$. No quantum algorithm can distinguish $\operatorname{SR}_{r}^{D_{i}}(\mathcal{X})$ from $D_{i}^{\mathcal{X}}$ with probability greater than $\ell(q) / r=\epsilon / 4$. Thus, it must be that the quantity

$$
\left|\operatorname{Pr}_{O \leftarrow \mathrm{SR}_{r}^{D_{1}}(\mathcal{X})}\left[B^{|O\rangle}()=1\right]-\underset{O \leftarrow \mathrm{SR}_{r}^{D_{2}}(\mathcal{X})}{\operatorname{Pr}}\left[B^{|O\rangle}()=1\right]\right|
$$

is at least $\epsilon / 2$. We now define $r+1$ hybrids $H_{i}$ as follows: For $j=0, \ldots, i-1$, draw $y_{j}$ from $D_{1}$. For $j=i, \ldots, r-1$, draw $y_{j}$ from $D_{2}$. Then give $B$ the oracle $O$ where for each $x, O(x)$ is a randomly selected $y_{i} . H_{r}$ is the case where $O \leftarrow \mathrm{SR}_{r}^{D_{1}}$, and $H_{0}$ is the case where $O \leftarrow \mathrm{SR}_{r}^{D_{2}}$. Hence $H_{0}$ and $H_{r}$ are distinguished with probability at least $\epsilon / 2$. Let

$$
\epsilon_{i}=\underset{O \leftarrow H_{i+1}}{\operatorname{Pr}}\left[B^{|O\rangle}()=1\right]-\underset{O \leftarrow H_{i}}{\operatorname{Pr}}\left[B^{|O\rangle}()=1\right]
$$

be the probability that $B$ distinguishes $H_{i+1}$ from $H_{i}$. Then $\left|\sum_{i=1}^{r} \epsilon_{i}\right| \geq \epsilon / 2$.

We construct an algorithm $A$ that distinguishes between $D_{1}$ and $D_{2}$ with probability $\epsilon / 2 r$. $A$, on inputs $y$, does the following:

- Choose a random $i \in[r]$.

- Construct a random oracle $O_{0} \leftarrow[r]^{\mathcal{X}}$.

- Construct random oracles $O_{1} \leftarrow D_{1}^{\{0, \ldots, i-1\}}$ and $O_{2} \leftarrow D_{2}^{\{i+1, \ldots, r-1\}}$.

- construct the oracle $O$ where $O(x)$ is defined as follows:

- Compute $j=O_{0}(x)$.

- If $j=i$, output $y$.

- Otherwise, if $j<i$, output $O_{1}(j)$ and if $j>i$, output $O_{2}(j)$.

- Simulate $B$ with the oracle $O$, and output the output of $B$.

Let $A_{i}$ be the algorithm $A$ using $i$. If $y \leftarrow D_{1}, B$ sees hybrid $H_{i+1}$. If $y \leftarrow D_{2}, B$ sees $H_{i}$. Therefore, we have that

$$
\operatorname{Pr}_{y \leftarrow D_{1}}\left[A_{i}(y)=1\right]-\operatorname{Pr}_{y \leftarrow D_{2}}\left[A_{i}(y)=1\right]=\epsilon_{i} .
$$

Averaging over all $i$, we get that $A$ 's distinguishing probability is

$$
\left|\operatorname{Pr}_{y \leftarrow D_{1}}[A(y)=1]-\operatorname{Pr}_{y \leftarrow D_{2}}[A(y)=1]\right|=\left|\frac{1}{r} \sum_{i=1}^{r} \epsilon_{i}\right| \geq \frac{\epsilon}{2 r}=\frac{\epsilon^{2}}{8 \ell(q)} .
$$

Thus, $A$ is an (efficient) algorithm that distinguishes $D_{1}$ from $D_{2}$ with non-negligible probability. Hence, it breaks the indistinguishability of $D_{1}$ and $D_{2}$.

Notice that by removing the requirement that $B$ be an efficient algorithm, we get a proof for the statistical setting as well, so that if any computationally unbounded quantum algorithm making $q$ quantum queries distinguishes $D_{1}^{\mathcal{X}}$ from $D_{2}^{\mathcal{X}}$ with probability $\epsilon$, then $D_{1}$ and $D_{2}$ must be $\Omega\left(\epsilon^{2} / \ell(q)\right)=\Omega\left(\epsilon^{2} / q^{3}\right)$-far, improving the result of Boneh et al. by a factor of $q$.

Now that Theorem 1.1 is proved, we have completed the proof of security for the GGM construction (Construction 1) in the quantum setting. With some modifications to the proof, we can also prove the quantum security for Constructions 2 and 3, as shown in Appendix E and Appendix F. 


\section{Conclusion}

We have shown that not all pseudorandom functions secure against classical queries are also secure against quantum queries. Nevertheless, we demonstrate the security of several constructions of pseudorandom functions against quantum queries. Specifically, we show that the construction from pseudorandom generators [GGM86], the construction from pseudorandom synthesizers [NR95], and the direct construction based on the Learning With Errors problem [BPR11] are all secure against quantum algorithms making quantum queries. We accomplish these results by providing more tools for bounding the ability of a quantum algorithm to distinguish between two oracle distributions. We leave as an open problem proving the quantum security of some classical uses of pseudorandom functions. We have two specific instances in mind:

- Pseudorandom permutations (Block Ciphers) secure against quantum queries. We know how to build pseudorandom permutations from pseudorandom functions in the classical setting ([LR88, NR99]). Classically, the first step to prove security is to replace the pseudorandom functions with truly random functions, which no efficient algorithm can detect. The second step is to prove that no algorithm can distinguish this case from a truly random permutation. For this construction to be secure against quantum queries, a quantum-secure pseudorandom function is clearly needed. However, it is not clear how to transform the second step of the proof to handle quantum queries.

- Message Authentication Codes (MACs) secure against quantum queries. MACs can be built from pseudorandom functions and proven existentially unforgeable against a classical adaptive chosen message attack. If we allow the adversary to ask for an authentication on a superposition of messages, a new notion of security is required. One possible definition of security is that, after $q$ queries, no adversary can produce $q+1$ classical valid message/tag pairs. Given a pseudorandom function secure against quantum queries, proving this form of security reduces to proving the impossibility of the following: After $q$ quantum queries to a random oracle $O$, output $q+1$ input/output pairs of $O$ with non-negligible probability.

\section{Acknowledgments}

We would like to thank Dan Boneh for his guidance and many insightful discussions. This work was supported by NSF and DARPA. Any opinions, findings, and conclusions or recommendations expressed in this material are those of the author(s) and do not necessarily reflect the views of the Department of Defense or the U.S. Government.

\section{A Proof of the Separation Result}

Here we finish the counter-example from Section 3 and the proof of Theorem 3.1 by proving Claims 1 and 2. Recall that we start with a pseudorandom function PRF with key-space $\mathcal{K}$, domain $[N]$, and range $\mathcal{Y}$ where $|\mathcal{Y}| \geq N^{2}$. We then construct a new pseudorandom function PRF $^{\prime}$ whose keys are pairs $(k, a)$ where $k \in \mathcal{K}$ and $a \in \mathcal{A}$ where $\mathcal{A}$ is the set of primes in $(N / 2, N]$. We let $N^{\prime}$ be the smallest power of 2 greater than $4 N^{2}$, and for $x \in\left[N^{\prime}\right]$, define $\operatorname{PRF}_{(k, a)}^{\prime}(x)=\operatorname{PRF}_{k}(x \bmod a)$. 
Proof of Claim 1. We prove that if PRF is standard-secure, so is PRF'. Suppose we have a quantum adversary $A$ making classical queries that distinguishes PRF $^{\prime}$ from a random function with non-negligible probability $\epsilon$. That is,

$$
\left|\operatorname{Pr}_{k \leftarrow \mathcal{K}, a \leftarrow \mathcal{A}}\left[A^{\mathrm{PRF}^{\prime}{ }_{(k, a)}}()=1\right]-\operatorname{Pr}_{O \leftarrow \mathcal{Y} \mathcal{X}}\left[A^{O}()=1\right]\right|=\epsilon
$$

This is equivalent to

$$
\left|\operatorname{Pr}_{k \leftarrow \mathcal{K}, a \leftarrow \mathcal{A}}\left[A^{\mathrm{PRF}_{k}(\cdot \bmod a)}()=1\right]-\operatorname{Pr}_{O \leftarrow \mathcal{Y}^{\mathcal{X}}}\left[A^{O}()=1\right]\right|=\epsilon
$$

Consider the quantity

$$
\left|\operatorname{Pr}_{O \leftarrow \mathcal{Y}^{\mathcal{X}}, a \leftarrow \mathcal{A}}\left[A^{O(\cdot \bmod a)}()=1\right]-\operatorname{Pr}_{O \leftarrow \mathcal{Y}^{\mathcal{X}}}\left[A^{O}()=1\right]\right|
$$

The left hand side is the case where $O$ is a random function in $\mathcal{Y}^{\mathcal{X}}, a$ is a random prime in $(N / 2, N]$, and we give $A$ the oracle $O^{\prime}(x)=O(x \bmod a)$. As long as $A$ never queries its oracle on two points $x$ and $x^{\prime}$ such that $x \equiv x^{\prime} \bmod a$, this oracle will look random. If $A$ makes $q$ queries, there are $\left(\begin{array}{l}q \\ 2\end{array}\right)$ possible differences between query points. Each difference is at most $8 N^{2}$, so for large $N$ it can only be divisible by at most 2 different moduli $a$. Notice that $|\mathcal{A}| \in \Omega(N / \log N)$. Each difference thus has a probability at most $2 /|\mathcal{A}| \in O(2 \log N / N)$ of being divisible by $a$, so the total probability of querying $x$ and $x^{\prime}$ such that $x \equiv x^{\prime} \bmod a$ is at most $O\left(q^{2} \log N / N\right)$. Thus this probability, and hence the ability of $A$ to distinguish $O^{\prime}$ from a random oracle, is negligible.

A simple hybrid argument then shows that

$$
\left|\operatorname{Pr}_{k \leftarrow \mathcal{K}, a \leftarrow \mathcal{A}}\left[A^{\mathrm{PRF}_{k}(\cdot \bmod a)}()=1\right]-\operatorname{Pr}_{O \leftarrow \mathcal{Y}^{\mathcal{X}}, a \leftarrow \mathcal{A}}\left[A^{O(\cdot \bmod a)}()=1\right]\right| \geq \epsilon-O\left(q^{2} \log N / N\right)
$$

Define a quantum algorithm $B$ which distinguishes PRF from a random oracle. $B$ has an oracle $O$, chooses a random prime $a \in(N / 2, N]$, and simulates $A$ with the oracle $O^{\prime}(x)=O(x \bmod a)$. When $O=\mathrm{PRF}_{k}$, we get the left side, and when $O$ is random, we get the right side. Thus,

$$
\left|\operatorname{Pr}_{k \leftarrow \mathcal{K}}\left[B^{\mathrm{PRF}_{k}}()=1\right]-\operatorname{Pr}_{O \leftarrow \mathcal{Y}}\left[B^{O}()=1\right]\right| \geq \epsilon-O\left(q^{2} \log N / N\right)
$$

Since $N$ is exponential, $B$ breaks the standard-security of PRF.

Proof of Claim 2. We now show that PRF and $\mathrm{PRF}^{\prime}$ cannot both be quantum-secure. Suppose $\mathrm{PRF}$ is quantum secure. We first consider the case where $\mathrm{PRF}^{\prime}$ is built from a truly random function $O:[N] \rightarrow \mathcal{Y}$. That is, $\operatorname{PRF}_{a}^{\prime}(x)=O(x \bmod a)$.

Since $|\mathcal{Y}| \geq N^{2}$, the probability that there is a collision $\left(x, x^{\prime}\right)$ where $O(x)=O\left(x^{\prime}\right)$ is less than $1 / 2$. In this case, we then notice that $\mathrm{PRF}^{\prime}$ is periodic with period $a$, and we can use the results of Boneh and Lipton [BL95] to find this period in polynomial time by making quantum queries to the oracle. Thus, we get a distinguisher that works as follows, given access to an oracle $O^{\prime}$ :

- Use the period-finding algorithm of Boneh and Lipton to find the period $a$ of $O^{\prime}$. 
- If $a \in(N / 2, N]$, pick a random $x \in\left[N^{\prime}-a\right]$, and verify that $O^{\prime}(x)=O^{\prime}(x+a)$. If so, output 1. Otherwise, output 0.

If $O^{\prime}=O(x \bmod a)$, then with probability at least $1 / 2, O$ will have no collisions, meaning we will find $a$ with probability $1-o(1) \cdot O^{\prime}(x)=O^{\prime}(x+a)$ will always be true in this case, so we output 1. If $O^{\prime}$ is random, then for any $x$, the probability that there is any $x^{\prime} \in x+(N / 2, N]$ with $O^{\prime}\left(x^{\prime}\right)=O^{\prime}(x)$ is negligible, so the random oracle will fail the test with all but negligible probability. Therefore, we distinguish $\mathrm{PRF}^{\prime}$ from random with probability at least $1 / 2-o(1)$.

We now switch to the true definition of $\mathrm{PRF}^{\prime}$. That is, we replace the random oracle $O$ with PRF. Since PRF is quantum-secure, this only affects the behavior of our distinguisher negligibly. Therefore, our distinguisher still distinguishes $\mathrm{PRF}^{\prime}$ from random with probability at least $1 / 2-o(1)$.

\section{B Proof of Theorem 7.3}

Here we prove Theorem 7.3. We will actually prove a more general version. Recall that we have a family of distributions $E_{r}$ over $\mathcal{Y}^{\mathcal{X}}$ parametrized by $r \in \mathbb{Z}^{+} \bigcup\{\infty\}$. For any $2 q$ pairs $\left(x_{i}, r_{i}\right)$, suppose the function $p(\lambda)=\operatorname{Pr}_{H \leftarrow E_{1 / \lambda}}\left[H\left(x_{i}\right)=r_{i} \forall i \in\{1, \ldots, 2 q\}\right]$ satisfies:

- $p$ is represented by a polynomial in $\lambda$ of degree at most $d$.

- $p^{(i)}(0)$, the $i$ th derivative of $p$ at 0 , is 0 for each $i \in\{1, \ldots, \Delta-1\}$.

Setting $\Delta=1$ gives the conditions of the theorem.

We will show that any $q$ query quantum algorithm can only distinguish $E_{r}$ from $E_{\infty}$ with probability at most $2^{2-\Delta} \zeta(2 \Delta)(1 / r)^{\Delta}(d)^{3 \Delta}$.

Let $\lambda=1 / r$. We follow the same proof technique as in Zhandry [Zha12].

By Theorem 7.1, for a q-query quantum algorithm $A, \operatorname{Pr}_{H \leftarrow E_{r}}\left[A^{H}()=z\right]$ is a linear combination of the $\operatorname{Pr}_{H \leftarrow E_{r}}\left[H\left(x_{i}\right)=r_{i} \forall i \in\{1, \ldots, 2 q\}\right]$. Thus, for any $z, \operatorname{Pr}_{H \leftarrow E_{1 / \lambda}}\left[A^{H}()=z\right]$ is a polynomial in $\lambda$ of degree $d$ with the first $\Delta-1$ derivatives at $\lambda=0$ being 0 .

Now, suppose that $A$ distinguishes $E_{1 / \lambda}$ from $E_{\infty}$ with probability $\epsilon(\lambda)$. That is

$$
\sum_{z}\left|\operatorname{Pr}_{H \leftarrow E_{1 / \lambda}}\left[A^{H}()=z\right]-\operatorname{Pr}_{H \leftarrow E_{\infty}}\left[A^{H}()=z\right]\right|=\epsilon(\lambda) .
$$

Let $\mathcal{Z}_{\lambda}$ be the set of $z$ such that $z$ is a more likely output under $E_{1 / \lambda}$ than $E_{\infty}$. That is, $\operatorname{Pr}_{H \leftarrow E_{1 / \lambda}}\left[A^{H}()=z\right]>\operatorname{Pr}_{H \leftarrow E_{\infty}}\left[A^{H}()=z\right]$. It is not difficult to show that

$$
\operatorname{Pr}_{H \leftarrow E_{1 / \lambda}}\left[A^{H}() \in \mathcal{Z}_{\lambda}\right]-\operatorname{Pr}_{H \leftarrow E_{\infty}}\left[A^{H}() \in \mathcal{Z}_{\lambda}\right]=\epsilon(\lambda) / 2 .
$$

Fix $\lambda_{0}$, and consider the quantity

$$
p_{\lambda_{0}}(\lambda) \equiv \operatorname{Pr}_{H \leftarrow E_{1 / \lambda}}\left[A^{H}() \in \mathcal{Z}_{\lambda_{0}}\right]=\sum_{z \in \mathcal{Z}_{\lambda_{0}}} \operatorname{Pr}_{H \leftarrow E_{1 / \lambda}}\left[A^{H}()=z\right] .
$$

Then $p_{\lambda}(\lambda)-p_{\lambda}(0)=\epsilon(\lambda) / 2$. Further, for each $\lambda_{0}, p_{\lambda_{0}}$ is a degree-d polynomial in $\lambda$ such that $p_{\lambda_{0}}^{(i)}(0)=0$ for $i \in\{1, \ldots, \Delta-1\}$. It also lies in the range $[0,1]$ when $\lambda=0$ or $1 / \lambda \in \mathbb{Z}^{+}$. Thus, we make use of the following theorem: 
Theorem B.1. Let $p(\lambda)$ be a polynomial in $\lambda$ of degree $d$ such that $p^{(i)}(0)=0$ for $i \in\{1, \ldots, \Delta-1\}$, $0 \leq p(0) \leq 1$, and $0 \leq p(1 / r) \leq 1$ for all $r \in \mathbb{Z}^{+}$. Then $|p(1 / r)-p(0)|<2^{1-\Delta} \zeta(2 \Delta)(1 / r)^{\Delta} d^{3 \Delta}$ for all $r \in \mathbb{Z}^{+}$, where $\zeta$ is the Riemann Zeta function.

Before proving this theorem, we use it to finish the proof of Theorem 7.3. For each $\lambda_{0}, p_{\lambda_{0}}$ satisfies the conditions of Theorem B.1, so we must have that $p_{\lambda_{0}}(\lambda)-p_{\lambda_{0}}(0)<2^{1-\Delta} \zeta(2 \Delta) \lambda^{\Delta} d^{3 \Delta}$. But then setting $\lambda_{0}=\lambda$, we get that

$$
\epsilon(\lambda)=2\left(p_{\lambda}(\lambda)-p_{\lambda}(0)\right)<2^{2-\Delta} \zeta(2 \Delta) \lambda^{\Delta} d^{3 \Delta} .
$$

Replacing $1 / \lambda$ with $r$, we have shown that the output distributions of any $q$ query quantum algorithm $A$ under $E_{r}$ and $E_{\infty}$ are $2^{2-\Delta} \zeta(2 \Delta)(1 / r)^{\Delta} d^{3 \Delta}$-close, as desired.

Proof of Theorem B.1. We have a polynomial $p$ of degree $d$ with $p^{(i)}(0)=0$ for $i \in\{1, \ldots, \Delta-1\}$. Further, for $r \in \mathbb{Z}^{+} \cap\{\infty\}, 0 \leq p(1 / r) \leq 1$. Now, let $s(\lambda)=\frac{p(\lambda)-p(0)}{\lambda^{\Delta}}$. Then $s$ is a $d-\Delta$-degree polynomial. We will now interpolate this polynomial at $d-\Delta+1$ points: let

$$
\lambda_{i}=\frac{1}{\left\lfloor\frac{(d-\Delta+1)^{3}}{2 i^{2}}\right\rfloor} .
$$

Then we can use the Lagrange interpolating polynomials to interpolate $s(\lambda)$. Let $s_{i}=s\left(\lambda_{i}\right)$. Then:

$$
s(\lambda)=\sum_{i=1}^{d-\Delta+1} s_{i} \ell_{i}(\lambda)
$$

where $\ell_{i}(\lambda)$ is the Lagrange polynomial

$$
\ell_{i}(\lambda)=\prod_{j=1, j \neq i}^{d-\Delta+1}\left(\frac{\lambda-\lambda_{j}}{\lambda_{i}-\lambda_{j}}\right)
$$

Then we get

$$
\begin{aligned}
p(\lambda)-p(0) & =\lambda^{\Delta} \sum_{i=1}^{d-\Delta+1} \frac{p\left(\lambda_{i}\right)-p(0)}{\lambda_{i}^{\Delta}} \ell_{i}(\lambda) \\
& =\sum_{i-1}^{d-\Delta+1} a_{i}(\lambda)\left(p\left(\lambda_{i}\right)-p(0)\right)
\end{aligned}
$$

where

$$
a_{i}(\lambda)=\left(\frac{\lambda}{\lambda_{i}}\right)^{\Delta} \ell_{i}(\lambda)
$$

Now, observe that $1 / \lambda_{i}$ are integers, so $0 \leq p\left(\lambda_{i}\right) \leq 1$ by assumption. Since $0 \leq p(0) \leq 1$ as well, we must have that $\left|p\left(\lambda_{i}\right)-p(0)\right| \leq 1$. Therefore,

$$
|p(\lambda)-p(0)|=\sum_{i=1}^{d-\Delta+1}\left|a_{i}(\lambda)\right| .
$$

We now need to bound this sum. 
Claim 3. If $\lambda \leq \lambda_{i}$ for all $i$, then $\sum_{i}\left|a_{i}(\lambda)\right|<2^{1-\Delta} \zeta(2 \Delta) \lambda^{\Delta} d^{3 \Delta}$

Before proving this claim, we note that it proves Theorem B.1 when $\lambda \leq \lambda_{i}$ for all $i$ (equivalently, $\left.\lambda \leq \lambda_{1}\right)$. If $\lambda>\lambda_{1}$, then the bound we are trying to prove is at least

$$
2^{1-\Delta} \zeta(2 \Delta)\left(\frac{(d-\Delta+1)^{3}}{\left\lfloor(d-\Delta+1)^{3} / 2\right\rfloor}\right)^{\Delta}>2 \zeta(2 \Delta)>2 .
$$

Which is already trivially satisfied by the assumption that $p(1 / r) \in[0,1]$.

Proof of Claim 3. First, notice that

$$
\left|\frac{a_{i}(\lambda)}{\lambda^{\Delta}}\right|=\left(\frac{1}{\lambda_{i}}\right)^{\Delta} \prod_{j=1, j \neq i}^{d-\Delta+1}\left(\frac{\left|\lambda-\lambda_{j}\right|}{\left|\lambda_{i}-\lambda_{j}\right|}\right) \leq\left(\frac{1}{\lambda_{i}}\right)^{\Delta} \prod_{j=1, j \neq i}^{d-\Delta+1}\left(\frac{\lambda_{j}}{\left|\lambda_{i}-\lambda_{j}\right|}\right)
$$

Now, observe that $\lambda_{i} \geq \frac{2 i^{2}}{(d-\Delta+1)^{3}}$ and that

$$
\begin{aligned}
\left|\lambda_{i}-\lambda_{j}\right| & =\lambda_{i} \lambda_{j}\left|\left\lfloor\frac{(d-\Delta+1)^{3}}{2 i^{2}}\right\rfloor-\left\lfloor\frac{(d-\Delta+1)}{2 j^{2}}\right\rfloor\right| \\
& \geq \frac{2 i^{2}}{(d-\Delta+1)^{3}} \lambda_{j}\left(\left|\frac{(d-\Delta+1)^{3}}{2 i^{2}}-\frac{(d-\Delta+1)^{3}}{2 j^{2}}\right|-1\right)
\end{aligned}
$$

Which can be simplified to

$$
\left|\lambda_{i}-\lambda_{j}\right| \geq \lambda_{j} \frac{\left|i^{2}-j^{2}\right|-\frac{2 i^{2} j^{2}}{(d-\Delta+1)^{3}}}{j^{2}}
$$

We notice that the numerator is minimized by making $i$ and $j$ as large as possible, which is when they are $d-\Delta+1$ and $d-\Delta$. In this case, the quantity becomes $\lambda_{j}\left(3-\frac{2}{d-\Delta+1}\right) / j^{2}$, which is greater than 0 as long as $d-\Delta+1 \geq 1$ (if $d-\Delta<0$, then $p(\lambda)$ is a constant, so the theorem is trivial).

Thus

$$
\left|\frac{a_{i}(\lambda)}{\lambda^{\Delta}}\right| \leq\left(\frac{1}{\lambda_{i}}\right)^{\Delta} \prod_{j=1, j \neq i}^{d-\Delta+1}\left(\frac{j^{2}}{\left|i^{2}-j^{2}\right|-\frac{2 i^{2} j^{2}}{(d-\Delta+1)^{3}}}\right)
$$

The $\left(1 / \lambda_{i}\right)^{\Delta}$ term is bounded by $\left(\frac{(d-\Delta+1)^{3}}{2 i^{2}}\right)^{\Delta}$. We now bound the other term:

Claim 4. For all integers $D$ and $i$ such that $i \leq D, \alpha_{D, i} \leq 2$ where

$$
\alpha_{D, i}=\prod_{j=1, j \neq i}^{D}\left(\frac{j^{2}}{\left|i^{2}-j^{2}\right|-\frac{2 i^{2} j^{2}}{D^{3}}}\right)
$$

Proof. First, rewrite $\alpha_{D, i}$ as

$$
\alpha_{D, i}=\prod_{j=1, j \neq i}^{D} \frac{j^{2}}{\left|i^{2}-j^{2}\right|} \prod_{j=1, j \neq i}^{D} \frac{1}{1-\frac{2 i^{2} j^{2}}{\left|i^{2}-j^{2}\right| D^{3}}}
$$


The first term is

$$
\begin{aligned}
\prod_{j=1, j \neq i}^{D} \frac{j^{2}}{\left|i^{2}-j^{2}\right|} & =\prod_{j=1}^{i-1} \frac{j^{2}}{(i+j)(i-j)} \prod_{j=i+1}^{D} \frac{j^{2}}{(i+j)(j-i)} \\
& =\left(\frac{((i-1) !)^{2}}{\frac{(2 i-1) !}{i !}(i-1) !}\right)\left(\frac{\left(\frac{D !}{i !}\right)^{2}}{\frac{(D+i) !}{(2 i) !}(D-i) !}\right) \\
& =\left(\frac{2(i !)^{2}}{(2 i) !}\right)\left(\frac{(D !)^{2}(2 i) !}{(i !)^{2}(D-i) !(D+i) !}\right) \\
& =2(D !)^{2} /(D-i) !(D+i) ! \\
& =\frac{\left(\begin{array}{c}
2 D \\
D+i
\end{array}\right)}{\left(\begin{array}{c}
2 D \\
D
\end{array}\right)}
\end{aligned}
$$

We use the fact that

$$
\left(\frac{d}{d k}\right)^{m} \log \left(\begin{array}{l}
n \\
k
\end{array}\right)=\psi^{(m-1)}(k+1)-(-1)^{m} \psi^{(m-1)}(n-k+1)
$$

Where $\psi^{(\ell)}(x)=\left(\frac{d}{d x}\right)^{\ell} \log \Gamma(x)$ is the polygamma function. This allows us to write:

$$
\frac{\left(\begin{array}{c}
2 D \\
D+i
\end{array}\right)}{\left(\begin{array}{c}
2 D \\
D
\end{array}\right)}=e^{-2 \sum_{\ell=1}^{\infty} \psi^{(2 \ell-1)}(d+1) \frac{i^{2 \ell}}{(2 \ell) !}}
$$

Next, we use the fact that $\psi^{(m)}(x) \geq 0$ for all odd $m$ and non-negative $x$, and $\psi^{(1)}(x+1) \leq \frac{1}{x}-\frac{1}{2 x^{2}}$ to bound

$$
\frac{\left(\begin{array}{c}
2 D \\
D+i
\end{array}\right)}{\left(\begin{array}{c}
2 D \\
D
\end{array}\right)} \leq e^{-\psi^{(1)}(d+1) i^{2} / 2} \leq e^{-i^{2}\left(\frac{1}{d}-\frac{1}{2 d^{2}}\right)}
$$

We now bound the second term

$$
\beta_{D, i}:=\prod_{j=1, j \neq i}^{D} \frac{1}{1-\frac{2 i^{2} j^{2}}{\left|i^{2}-j^{2}\right| D^{3}}}
$$

First, let $x_{i, j}=\frac{2 i^{2} j^{2}}{\left|i^{2}-j^{2}\right| D^{3}}$. Notice that $x_{i, j}=x_{j, i}$, and that for $j<i, x_{i, j} \leq x_{i, i-1}$. Lastly, notice that $x_{i, i-1} \leq x_{D, D-1}$. Therefore, for all $i, j$,

$$
x_{i, j} \leq \frac{2(D-1)^{2}}{D(2 D-1)} \leq 1-\frac{1}{D}
$$

Now, we use the fact that $\frac{1}{1-x} \leq e^{x+x^{2} \ln D}$ for all $x \leq 1-1 / D$ to bound

$$
\ln \beta_{D, i} \leq \sum_{j=1, j \neq i}^{D} x_{i, j}+x_{i, j}^{2} \ln D
$$


Much the same way as we analyzed the first term by breaking the product into cases where $j<i$ and $j>i$, we can do the same for this sum. To simplify notation, we use the generalized harmonic numbers:

$$
H_{n}^{(r)}=\sum_{j=1}^{n} \frac{1}{j^{r}}
$$

and $H_{n}=H_{n}^{(1)}$. Evaluating the sum in terms of the harmonic number, we can write

$$
\begin{aligned}
\ln \beta_{D, i} \leq & \frac{i^{2}}{2 D^{3}}\left(1+4 D-8 i+2 i\left(H_{D-i}-H_{D+i}+2 H_{2 i}\right)\right) \\
& +\frac{i^{4} \ln D}{4 D^{6}}\left(-3+16 D+12 i\left(H_{D-i}-H_{D+i}\right)+4 i^{2}\left(H_{D-i}^{(2)}+H_{D+i}^{(2)}\right)\right)
\end{aligned}
$$

Putting together with the first part, we have that

$$
\begin{aligned}
\ln \left(\frac{\alpha_{D, i}}{2}\right) \leq & \frac{i^{2}}{2 D^{3}}\left(1+5 D-2 D^{2}-8 i+2 i\left(H_{D-i}-H_{D+i}+2 H_{2 i}\right)\right) \\
& +\frac{i^{4} \ln D}{4 D^{6}}\left(-3+16 D+12 i\left(H_{D-i}-H_{D+i}\right)+4 i^{2}\left(H_{D-i}^{(2)}+H_{D+i}^{(2)}\right)\right) \\
= & \frac{i^{2}}{4 D^{6}}\left[-4 D^{5}+\left(4 D^{3} i\left(H_{D-i}-H_{D+i}+2 H_{2 i}\right)+10 D^{4}-16 D^{3} i+4 i^{4}\left(H_{D-i}^{(2)}+H_{D+i}^{(2)}\right) \ln D\right)\right. \\
& \left.+\left(2 D^{3}+16 D i^{2} \ln D+12 i^{3}\left(H_{D-i}-H_{D+i}\right) \ln D\right)-3 i^{2} \ln D\right]
\end{aligned}
$$

In order to prove the claim, we need to show that the quantity on the left is less than 0 . We can already see that, for large enough $D$, since $1 \leq D \leq i$ and $H_{n}=O(\log n)$ and $H_{n}^{(2)}=O(1)$, that the $-4 D^{5}$ term will dominate, and therefore the whole quantity will indeed be less than 0 . It remains to find what $D$ suffices.

Let's first look at the quartic term (where powers of $i$ and $D$ sum to 4 ):

$$
4 D^{3} i\left(H_{D-i}-H_{D+i}+2 H_{2 i}\right)+10 D^{4}-16 D^{3} i+4 i^{4}\left(H_{D-i}^{(2)}+H_{D+i}^{(2)}\right) \ln D
$$

Let $r=i / D$. We use the fact that, $\log n \leq H_{n} \leq \log n+1$ and $H_{n}^{(2)} \leq \pi^{2} / 6$ to bound this above by:

$$
D^{4}\left(4 r(3+\ln (1-r)-\ln (1+r)+2 \ln (2 r)+2 \ln (2 D))+10-16 r+\frac{4}{3} \pi^{2} r^{4} \ln D\right)
$$

Next, we use the fact that $\ln 2 \leq 3 / 4$ to bound this as

$$
D^{4}\left(4 r\left(2+\ln \left(\frac{(1-r) r^{2}}{1+r}\right)+2 \ln (D)\right)+10+\frac{4}{3} \pi^{2} r^{4} \ln D\right)
$$

In the range $r \in[0,1],(1-r) r^{2} /(1+r)$ obtains a maximum value of $(5 \sqrt{5}-11) / 2<0.1$. This means $\ln \left(\frac{(1-r) r^{2}}{1+r}\right)<\ln (0.1)<-2$. Therefore, we upper bound the quantity as

$$
D^{4}\left(\left(8+\frac{4}{3} \pi^{2}\right) \ln D+10\right) \leq D^{4}(22 \ln D+10)
$$

We now bound the cubic term

$2 D^{3}+16 D i^{2} \ln D+12 i^{3}\left(H_{D-i}-H_{D+i}\right) \ln D \leq D^{3}\left(2+16 r^{2} \ln D+12 r^{3}(1+\ln (1-r)-\ln (1+r)) \ln D\right)$ 
Now, $1+\ln (1-r)-\ln (1+r) \leq 1-2 r$, allowing us to bound this as

$$
2 D^{3}\left(1+4 r^{2}\left(2+3 r-6 r^{2}\right) \ln D\right)
$$

$r^{2}\left(2+3 r-6 r^{2}\right)$ obtains a maximum value less than $3 / 4$, meaning we can bound this even further as $2 D^{3}(1+3 \ln D)$.

Putting this all together, we have that

$$
\ln \left(\frac{\alpha_{D, i}}{2}\right) \leq \frac{r^{2}}{4 D^{2}}\left(-4 D^{3}+D^{2}(22 \ln D+10)+2 D(3 \ln D+1)\right)
$$

The function $-4 D^{4}+D^{2}(22 \ln D+10)+2 D(3 \ln D+1)$ is less than 0 for all $D \geq 19$. Therefore, we have proved $\alpha_{D, i} \leq 2$ in this range. The remaining 171 cases for $D<19$ can easily (though tediously) be verified manually. This completes the proof of the claim.

With this proved, we can now complete the proof of Claim 3.

$$
\left|\frac{a_{i}(\lambda)}{\lambda^{\Delta}}\right| \leq\left(\frac{1}{\lambda_{i}}\right)^{\Delta+1} \prod_{j=1, j \neq i}^{d-\Delta+1}\left(\frac{j^{2}}{\left|i^{2}-j^{2}\right|-\frac{2 i^{2} j^{2}}{(d-\Delta+1)^{3}}}\right) \leq\left(\frac{(d-\Delta+1)^{3}}{2 i^{2}}\right)^{\Delta} \times 2 \leq 2\left(\frac{d^{3}}{2 i^{2}}\right)^{\Delta}
$$

This gives

$$
\left|a_{i}(\lambda)\right| \leq \lambda^{\Delta} d^{3 \Delta} 2^{1-\Delta} \frac{1}{i^{2 \Delta}}
$$

Summing over all $i$ from 1 to $d-\Delta+1$ gives

$$
\sum_{i=1}^{d-\Delta+1}\left|a_{i}(\lambda)\right| \leq \lambda^{\Delta} d^{3 \Delta} 2^{1-\Delta} \sum_{i=1}^{d-\Delta+1} \frac{1}{i^{2 \Delta}}
$$

The sum on the right hand side is the truncated $p$ series for $p=2 \Delta$. This series sums to $\zeta(p)$ where $\zeta$ is the Riemann Zeta function, so the truncation is strictly less than this value. Therefore,

$$
\sum_{i=1}^{d-\Delta+1}\left|a_{i}(\lambda)\right|<\lambda^{\Delta} d^{3 \Delta} 2^{1-\Delta} \zeta(2 \Delta)
$$

\section{Proof of Lemma 7.4}

In this section, we prove Lemma 7.4.

Proof of Lemma 7.4. Our goal is to show that, for each of the marginal distributions over $k$ inputs to $\mathrm{SR}_{r}^{D}$, each probability is a polynomial in $1 / r$ of degree at most $k$.

Fix some $x_{i}$ and $y_{i}$ for $i \in[k]$. We consider the probability that $O\left(x_{i}\right)=y_{i}$ for all $i \in[k]$. We can assume without loss of generality that the $x_{i}$ are distinct. Otherwise, there are $i, j$ such that $x_{i}=x_{j}$. If $y_{i} \neq y_{j}$, then the probability is 0 ( $O$ is not a function in this case). If $y_{i}=y_{j}$, the $O\left(x_{j}\right)=y_{j}$ condition is redundant and can be removed, reducing this to the $k-1$ case. By induction on $k$, the resulting probability is a polynomial of degree at most $k-1<k$. 
Recall that $\mathrm{SR}_{r}^{D}=D^{[r]} \circ[r]^{\mathcal{X}}$ and $D$ is a distribution on $\mathcal{Y}$. Let $O_{1} \leftarrow[r]^{\mathcal{X}}$ and $O_{2} \leftarrow D^{[r]}$. Let $O_{1}^{\prime}$ be the restriction of $O_{1}$ to $\left\{x_{0}, \ldots, x_{k-1}\right\}$. Each $O_{1}^{\prime}$ then occurs with probability $1 / r^{k}$. Now,

$$
\begin{aligned}
& \underset{O \leftarrow \mathrm{SR}_{r}^{D}}{\operatorname{Pr}}\left[O\left(x_{i}\right)=y_{i} \forall i \in[k]\right]=\underset{O_{1} \leftarrow[r]^{\mathcal{X}}, O_{2} \leftarrow D^{[r]}}{\operatorname{Pr}}\left[O_{2}\left(O_{1}\left(x_{i}\right)\right)=y_{i} \forall i \in[k]\right]
\end{aligned}
$$

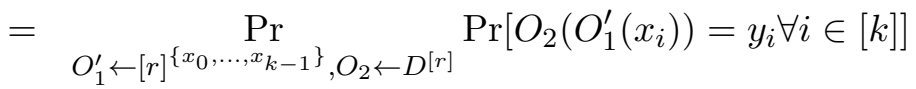

$$
\begin{aligned}
& =\frac{1}{r^{k}} \sum_{O_{1}^{\prime}} \underset{O_{2} \leftarrow D^{[r]}}{\operatorname{Pr}}\left[O_{2}\left(O_{1}^{\prime}\left(x_{i}\right)\right)=y_{i} \forall i \in[k]\right]
\end{aligned}
$$

We now associate with each $O_{1}^{\prime}$ a partition $P$ of $[k]$ into $r$ disjoint subsets $P_{j}$ for $j \in[r]$. The elements of $P_{j}$ are the indicies $i$ such that $O_{1}^{\prime}\left(x_{i}\right)=j$. Thus:

$$
\operatorname{Pr}_{O \leftarrow \mathrm{SR}_{r}^{D}}\left[O\left(x_{i}\right)=y_{i} \forall i \in[k]\right]=\frac{1}{r^{k}} \sum_{P=\left(P_{j}\right)} \operatorname{Pr}\left[O_{2}(j)=y_{i} \forall j \in[r], \forall i \in P_{j}\right]
$$

Since $\mathrm{O}_{2} \leftarrow D^{[r]}$, the distribution of outputs of $\mathrm{O}_{2}$ for each $j$ are independent. Thus the probabilities $\operatorname{Pr}\left[O_{2}(j)=y_{i} \forall i \in P_{j}\right]$ are also independently distributed. Thus,

$$
\underset{O \leftarrow \mathrm{SR}_{r}^{D}}{\operatorname{Pr}}\left[O\left(x_{i}\right)=y_{i} \forall i \in[k]\right]=\frac{1}{r^{k}} \sum_{P=\left(P_{j}\right)} \prod_{j \in[r]} \operatorname{Pr}\left[O_{2}(j)=y_{i} \forall i \in P_{j}\right]
$$

Since there are only $k$ elements, at most $k$ of the $P_{j}$ s are non-empty. Thus, we can associate to each partition $P$ another partition $Q$ of $[k]$ into $k_{Q} \leq k$ non-empty subsets, and a strictly increasing function from $f_{Q}$ from $\left[k_{Q}\right] \rightarrow[r]$. The association is as follows: $Q_{j^{\prime}}=P_{f_{Q}\left(j^{\prime}\right)}$ and $P_{j}=\emptyset$ if $j$ has no pre-image under $f_{Q}$. This allows us to write:

$$
\underset{O \leftarrow \mathrm{SR}_{r}^{D}}{\operatorname{Pr}}\left[O\left(x_{i}\right)=y_{i} \forall i \in[k]\right]=\frac{1}{r^{k}} \sum_{Q=\left(Q_{j^{\prime}}\right)} \sum_{f_{Q}} \prod_{j^{\prime} \in\left[k_{Q}\right]} \operatorname{Pr}\left[O_{2}\left(f_{Q}\left(j^{\prime}\right)\right)=y_{i} \forall i \in Q_{j^{\prime}}\right]
$$

We now notice that, for fixed $j^{\prime}$, if the $y_{i}$ are all equal for $i \in Q_{j^{\prime}}$, then since $O_{2} \leftarrow D^{[r]}$, $\operatorname{Pr}\left[O_{2}\left(f_{Q}\left(j^{\prime}\right)\right)=y_{i} \forall i \in Q_{j^{\prime}}\right]=D\left(y_{i}\right)$ where $i$ is any index in $Q_{j^{\prime}}$. Otherwise, $\operatorname{Pr}\left[O_{2}(j)=y_{i} \forall i \in\right.$ $\left.Q_{j^{\prime}}\right]=0$ since $O_{2}$ needs to be a function. Thus we can write $\operatorname{Pr}\left[O_{2}(j)=y_{i} \forall i \in Q_{j^{\prime}}\right]=D\left(y_{i}\right) \sigma\left(Q_{j^{\prime}}\right)$ where $\sigma(S)$ is 1 if $y_{i}$ are all equal for $i \in S$, and 0 otherwise. Thus,

$$
\underset{O \leftarrow \mathrm{SR}_{r}^{D}}{\operatorname{Pr}}\left[O\left(x_{i}\right)=y_{i} \forall i \in[k]\right]=\frac{1}{r^{k}} \sum_{Q=\left(Q_{j^{\prime}}\right)} \sum_{f_{Q}} \prod_{j^{\prime} \in\left[k_{Q}\right]} D\left(y_{i}\right) \sigma\left(Q_{j^{\prime}}\right)
$$

The summand does not depend on $f_{Q}$, so let $c_{Q}$ be the number of $f_{Q}$. Then we can write

$$
\underset{O \leftarrow \mathrm{SR}_{r}^{D}}{\operatorname{Pr}}\left[O\left(x_{i}\right)=y_{i} \forall i \in[k]\right]=\frac{1}{r^{k}} \sum_{Q=\left(Q_{j^{\prime}}\right)} c_{Q} \prod_{j^{\prime} \in\left[k_{Q}\right]} \operatorname{Pr}\left[O_{2}\left(j^{\prime}\right)=y_{i} \forall i \in Q_{j^{\prime}}\right]
$$

The $Q$ we are summing over are independent of $r$, as is the product in the above expression. $c_{Q}$ is equal to the number of ways of picking $k_{Q}$ distinct elements of $[r]$, which is $\left(\begin{array}{c}r \\ k_{Q}\end{array}\right)$, and is thus polynomial of degree $k_{Q}$ in $r$ (and hence a polynomial of degree at most $k$ ). Therefore, performing the sum, $\operatorname{Pr}_{O \leftarrow \mathrm{SR}_{r}^{D}}\left[O\left(x_{i}\right)=y_{i} \forall i \in[k]\right]$ is a polynomial of degree at most $k$ in $r$, divided by $r^{k}$. The result is a polynomial of degree at most $k$ in $1 / r$. 


\section{A Quantum Distinguisher for Small-Range Distributions}

In this section, we give a quantum distinguisher that distinguishes $\mathrm{SR}_{r}^{\mathcal{Y}}$ from a random function with probability (asymptotically) matching the bound of Corollary 7.5. Our algorithm is basically the collision finding algorithm of Brassard, Høer, and Tapp [BHT97], with a check at the end to verify that a collision is found. The algorithm has oracle access to a function $O$ from $\mathcal{X}$ to $\mathcal{Y}$, which is either $\mathrm{SR}_{r}^{\mathcal{Y}}$ or a random function. It is given as input the integer $r$, the number of queries $q$, and operates as follows:

- Let $p=(q-1) / 2$. Pick a set $S$ of $p$ points in $\mathcal{X}$ at random, and check that there is no collision on $S$ by making $p$ classical queries to $O$. Sort the elements of $S$, and store the pairs $(s, O(s))$ as a table for efficient lookup.

- Construct the oracle $O^{\prime}(x)= \begin{cases}1 & \text { if } x \notin S \text { and } O(x)=O(s) \text { for some } s \in S \\ 0 & \text { otherwise }\end{cases}$

- Run Grover's algorithm [Gro96] on $O^{\prime}$ for $p$ iterations to look for a point $x$ such that $O^{\prime}(x)=1$.

- Check that there is an $s \in S$ such that $O(x)=O(s)$ by making one more classical query to $O$.

Before analyzing this construction, we explain what Grover's algorithm does. It takes as input an oracle $O^{\prime}$ mapping some space $\mathcal{X}$ into [2], and tries to find an $x$ such that $O^{\prime}(x)=1$. Specifically, if $N$ points map to 1 , then after $q$ queries to $O^{\prime}$, Grover's algorithm will output an $x$ such that $O^{\prime}(x)=1$ with probability $\Theta\left(q^{2} N /|\mathcal{X}|\right)$

We now analyze this construction. The first step takes $p$ queries to $O$. If we find a collision, we are done. Otherwise, we have $p$ points that map to $p$ different values. Call this set of values $T$. The oracle $O^{\prime}$ outlined in the second step makes exactly one query to $O$ for each query to $O^{\prime}$. The number of points in $x$ such that $O^{\prime}(x)=1$ is the number of points $x$ in $\mathcal{X} \backslash S$ (which is $|X|-p)$ such that $O(x) \in T$. In the random oracle case, the probability that $O(x)$ is one of $p$ random values is $p /|\mathcal{Y}|$, so the expected number of such $x$ is $(|\mathcal{X}|-p) p /|\mathcal{Y}|$. Thus, after $p$ iterations, Grover's algorithm will output such an $x$ with probability $\left.\Theta\left(p^{3}(|\mathcal{X}|-p) /|\mathcal{X}||\mathcal{Y}|\right)\right)$. In the $\mathrm{SR}_{r}^{\mathcal{Y}}$ case, since there are only $r$ possible outputs, the probability that $x$ maps to $T$ is $p / r$, so the expected number of such $x$ is $p(|\mathcal{X}|-p) / r$. Thus, Grover's algorithm will output such an $x$ with probability $\Theta\left(p^{3}(|\mathcal{X}|-p) / r|\mathcal{X}|\right)$.

The difference in these probabilities is $\Theta\left(p^{3}(1 / r-1 /|\mathcal{Y}|)(|\mathcal{X}|-p) /|\mathcal{X}|\right.$. If we let $|\mathcal{Y}|$ be at least $2 r$ and $|\mathcal{X}|$ at least $2 p+1=q$, we see that we distinguish $\mathrm{SR}_{r}^{\mathcal{Y}}$ from random with probability $\Omega\left(p^{3} / r\right)=\Omega\left(q^{3} / r\right)$, thus matching the bound of Corollary 7.5. This shows that the corollary is optimal, and hence Theorem 7.3 is optimal for the case $\Delta=0$.

\section{E Security Proof for the Synthesizer-Based PRF}

Here, we prove Theorem 5.3 by showing that PRF from Construction 2 is quantum secure if the underlying synthesizer $S$ is standard-secure.

Recall the definition of standard-security for a synthesizer $S: \mathcal{X}^{2} \rightarrow \mathcal{Y}$ from Definition 5.2: for all sets $\mathcal{Z}$, no efficient quantum algorithm $A$ making classical queries to an oracle $O$ from $\mathcal{Z}^{2} \rightarrow \mathcal{Y}$ can tell if $O\left(z_{1}, z_{2}\right)=S\left(O_{1}\left(z_{1}\right), O_{2}\left(z_{2}\right)\right)$ for random oracles $O_{i} \leftarrow \mathcal{X}^{\mathcal{Z}}$ or if $O$ is truly random. 
Since all queries are classical, and only a polynomial number of queries are possible, a simple argument shows that Definition 5.2 is equivalent to the case where $|\mathcal{Z}| \in n^{O(1)}$. Further, if $\mathcal{Z}$ is polynomial in size, we can query the entire set classically, so there is no advantage in having quantum queries. Therefore, Definition 5.2 is equivalent to the following:

Definition E.1 (Standard-Security). A pseudoreandom synthesizer $S: \mathcal{X}^{2} \rightarrow \mathcal{Y}$ is standard-secure if, for any set $\mathcal{Z}$ where $|\mathcal{Z}| \in n^{O(1)}$, no efficient quantum algorithm $A$ making quantum queries can distinguish $O\left(z_{1}, z_{2}\right)=S\left(O_{1}\left(z_{1}\right), O_{2}\left(z_{2}\right)\right)$ where $O_{b} \leftarrow \mathcal{X}^{\mathcal{Z}}$ from $O \leftarrow \mathcal{Y}^{\mathcal{Z} \times \mathcal{Z}}$.

Before proving security, we define the quantum-security of a pseudorandom synthesizer. The definition is similar to Definition E.1, except that there is no bound on the size of $\mathcal{Z}$ :

Definition E.2 (Quantum-Security). A pseudoreandom synthesizer $S: \mathcal{X}^{2} \rightarrow \mathcal{Y}$ is quantumsecure if, for any set $\mathcal{Z}$, no efficient quantum algorithm $A$ making quantum queries can distinguish $O\left(z_{1}, z_{2}\right)=S\left(O_{1}\left(z_{1}\right), O_{2}\left(z_{2}\right)\right)$ where $O_{b} \leftarrow \mathcal{X}^{\mathcal{Z}}$ from $O \leftarrow \mathcal{Y}^{\mathcal{Z} \times \mathcal{Z}}$

We now show that the two definitions are equivalent:

Lemma E.3. If $S$ is standard-secure, then it is also quantum-secure.

Proof. Let's define a new oracle distribution, which we will denote $\mathrm{AR}_{s}$, which stands for almost random. $\mathrm{AR}_{s}$ is defined as follows:

- Pick random oracles $P_{1}$ and $P_{2}$ from $[s]^{\mathcal{Z}}$.

- Pick a random oracle $Q$ from $\mathcal{Y}^{[s]^{2}}$.

- Output the oracle $O\left(z_{1}, z_{2}\right)=Q\left(P_{1}\left(z_{1}\right), P_{2}\left(z_{2}\right)\right)$.

Notice that as $s$ goes to $\infty, O_{1}$ and $O_{2}$ become injective with probability approaching 1 , and thus $\mathrm{AR}_{\infty}$ is the uniform distribution.

Now, let $B$ be an adversary breaking the oracle-security of $S$ with non-negligible probability $\epsilon$. Define $\epsilon(s)$ as the following quantity:

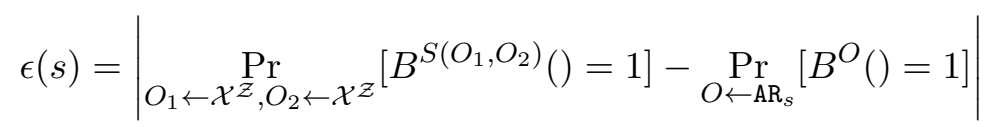

Then $\epsilon=\lim _{s \rightarrow \infty} \epsilon(s)$. Let $r$ be an integer such that $\ell(q) / r=\epsilon / 8$ where $q$ in the number of queries made by $B$. We now replace $O_{i}$ with $\mathrm{SR}_{r}^{\mathcal{X}}$, and the $P_{i}$ (as a part of $\mathrm{AR}_{s}$ ) with $\mathrm{SR}_{r}^{[s]}$. Each of these changes will only change the behavior of $A$ by $\epsilon / 8$. Thus, a simple argument shows that

$$
\left|\operatorname{Pr}_{O_{i} \leftarrow \mathrm{SR}_{r}^{\mathcal{X}}}\left[B^{S\left(O_{1}, O_{2}\right)}()=1\right]-\operatorname{Pr}_{P_{i} \leftarrow \mathrm{SR}_{r}^{[s]}, Q \leftarrow \mathcal{Y}[s]^{2}}\left[B^{Q\left(P_{1}, P_{2}\right)}()=1\right]\right| \geq \epsilon(s)-\epsilon / 2
$$

Notice that we can think of the oracle $Q\left(P_{1}, P_{2}\right)$ as the oracle

$$
O^{\prime}\left(z_{1}, z_{2}\right)=Q\left(S_{1} \circ R_{1}\left(z_{1}\right), S_{2} \circ R_{2}\left(z_{2}\right)\right)=O\left(R_{1}\left(z_{1}\right), R_{2}\left(z_{2}\right)\right)
$$

Where $S_{i} \leftarrow[s]^{[r]}, R_{i} \leftarrow[r]^{\mathcal{Z}}$, and $O\left(w_{1}, w_{2}\right)=Q\left(S_{1}\left(w_{1}\right), S_{2}\left(w_{2}\right)\right)$. As $s$ goes to $\infty, S_{i}$ become injective with probability converging to one, so $O$ approaches a random function from $[r]^{2} \rightarrow \mathcal{Y}$. 
We now describe a new algorithm $A$ which tries to break the standard-security of $S$ according to Definition E.1. $A$ takes as input a quantum-accessible oracle $O$ from $[r]^{2}$ to $\mathcal{Y}$. $A$ constructs two random oracles $R_{1} \leftarrow[r]^{\mathcal{Z}}$ and $R_{2} \leftarrow[r]^{\mathcal{Z}}$, gives $B$ the oracle $O^{\prime}\left(z_{1}, z_{2}\right)=O\left(R_{1}\left(z_{1}\right), R_{2}\left(z_{2}\right)\right)$, and simulates $B$. If $O=S\left(T_{1}, T_{2}\right)$ for random oracles $T_{i} \leftarrow \mathcal{X}^{[r]}$, then the oracle seen by $A$ is $O^{\prime}\left(z_{1}, z_{2}\right)=S\left(O_{1}\left(z_{1}\right), O_{2}\left(z_{2}\right)\right)$, where $O_{1}$ and $O_{2}$ are drawn from $\mathrm{SR}_{r}^{\mathcal{X}}$. If $O$ is a random oracle, then the oracle seen by $A$ is $O^{\prime}\left(z_{1}, z_{2}\right)=O\left(R_{1}\left(z_{1}\right), R_{2}\left(z_{2}\right)\right)$, where $R_{i} \leftarrow[r]^{\mathcal{Z}}$. This corresponds to the case where $s=\infty$, and thus the advantage of $A$ in distinguishing these two cases is $\epsilon(\infty)-\epsilon / 2=\epsilon / 2$. If $\epsilon$ is non-negligible, then there is a polynomial bounding $r$ infinitely often, and in these cases, $A$ breaks the standard-security of $S$.

We are now ready to prove that Construction 2 is quantum-secure:

Proof of Theorem 5.3. Let $A$ be a quantum adversary breaking the quantum-security of PRF with probability $\epsilon$. That is,

$$
\left|\operatorname{Pr}_{k \leftarrow \mathcal{X}^{2 n}}\left[A^{\mathrm{PRF}_{k}}()=1\right]-\underset{O \leftarrow \mathcal{X}^{[2]^{n}}}{\operatorname{Pr}}\left[A^{O}()=1\right]\right|=\epsilon
$$

Let hybrid $H_{i}$ be the game where the oracle seen by $A$ is PRF, except that each instance of $\operatorname{PRF}^{(i)}$ is replaced with a truly random function from $[2]^{2^{i}}$ into $\mathcal{X}$. Since $\mathrm{PRF}^{(0)}$ is already a random function, $H_{0}$ is equivalent to the case where the oracle is PRF. Similarly, $H_{\ell}$ is by definition the case where the oracle is truly random. Thus a simple hybrid argument shows that there is an $i$ such that $A$ can distinguish $H_{i}$ from $H_{i-1}$ with probability at least $\epsilon / \ell$.

We now describe an algorithm $B$ which breaks the quantum-security of $S$. $B$ is given an oracle from $P$ from $\left(\mathcal{X} \times\left[2^{\ell-i}\right]\right)^{2}$ into $\mathcal{X}$, which is either $S\left(Q_{1}, Q_{2}\right)$ for random oracles $Q_{b} \leftarrow \mathcal{X}^{\mathcal{X} \times\left[2^{\ell-i}\right]}$ or a truly random oracle. It then constructs oracles

$$
P_{y}\left(x_{1}, x_{2}\right)=P\left(\left(x_{1}, y\right),\left(x_{2}, y\right)\right)
$$

Notice that there are $2^{\ell-i}$ possible $y$ values, and that for fixed $y, P_{y}$ is either a random oracle from $\mathcal{X}^{2}$ into $\mathcal{X}$, or it is $S\left(Q_{y, 1}, Q_{y, 2}\right)$ for random oracles $Q_{y, b}$ from $\mathcal{X}$ to $\mathcal{X}$. We then construct the oracle $O$ which is PRF, except that we stop the recursive construction at $\operatorname{PRF}^{(i)}$. There are $2^{\ell-i}$ different instances of $\mathrm{PRF}^{(i)}$, so we use the $2^{\ell-i} P_{y}$ oracles in their place. If $P$ is $S\left(Q_{1}, Q_{2}\right)$, this corresponds to hybrid $H_{i-1}$, whereas if $P$ is a random oracle, this corresponds to $H_{i}$. Thus, $B$ distinguishes the two cases with probability $\epsilon / \ell$.

However, under the assumption that $S$ is standard-secure, Lemma E.3 shows that it is quantumsecure, meaning the algorithm $B$ is impossible. Therefore, PRF is quantum-secure.

\section{F Security Proof for the Direct Construction}

Here we give a precise statement and proof for Theorem 6.1, which state's that PRF from Construction 3 is quantum secure for the right parameters. First, we define the Learning With Errors (LWE) problem:

Definition F.1 (Learning With Errors). Let $q \geq 2$ an integer, $n$ a security parameter, and $m=\operatorname{poly}(n)$ and $w=\operatorname{poly}(n)$ be integers. For a distribution $\chi$ over $\mathbb{Z}$ and a secret matrix $\mathbf{S} \in \mathbb{Z}_{q}^{n \times w}$, the LWE distribution $\mathrm{LWE}_{\mathbf{S}, \chi}$ is the distribution over $\mathbb{Z}_{q}^{m \times n} \times \mathbb{Z}_{q}^{m \times w}$ defined as follows: 
- Choose a random matrix $\mathbf{A} \leftarrow \mathbb{Z}_{q}^{n \times m}$.

- Choose a random error matrix $\mathbf{E} \leftarrow \chi^{m \times w}$

- Output $\left(\mathbf{A}^{t}, \mathbf{B}^{t}=\mathbf{A}^{t} \mathbf{S}+\mathbf{E} \bmod q\right)$

The LWE problem is then to distinguish between a polynomial number of samples from $\mathrm{LWE}_{\mathbf{S}, \chi}$ for a fixed $\mathbf{S} \leftarrow \chi^{n \times w} \bmod q$ from the same number of samples from the uniform distribution. The LWE problem is hard if, for all efficient quantum adversaries $A$, the probability $A$ distinguishes these two cases is negligible in $n$.

We now define the oracle-LWE problem:

Definition F.2 (Oracle-LWE). The oracle-LWE problem is to distinguish an oracle $O$ whose outputs are generated by $\mathrm{LWE}_{\mathbf{S}, \chi}$ (for a fixed $\mathbf{S} \leftarrow \chi^{n \times w} \bmod q$ ) from a truly random oracle $O$. We say that LWE is oracle-hard if, for all efficient adversaries A making quantum queries, A cannot distinguish these two distributions with more than negligible probability.

Lemma F.3. If LWE is hard, it is also oracle-hard.

Proof. The proof is very similar to that of Theorem 1.1. Let $A$ be an adversary breaking the oracle-hardness of LWE using $q$ quantum queries with probability $\epsilon$. Let $r$ be an integer such that $\ell(q) / r \approx \epsilon / 4$. We then construct an algorithm $B$, which takes as input $r$ pairs $\left(\mathbf{A}_{i}^{t}, \mathbf{B}_{i}^{t}\right)$, and distinguishes when the pairs come from $\operatorname{LWE}_{\mathbf{S}, \chi}$ for some fixed $\mathbf{S} \leftarrow \chi^{n \times w}$ from when the pairs are random. $B$ works as follows:

- Construct the oracle $O$ where $O(x)$ is selected at random from $\left(\mathbf{A}_{i}^{t}, \mathbf{B}_{i}^{t}\right)$

- Simulate $A$ with oracle $O$, and output the output of $A$.

Using the same analysis as in the proof of Theorem 1.1, we get that $B$ distinguishes the two cases with probability $\epsilon / 2$. If $\epsilon$ is non-negligible, then there is a polynomial that bounds $r$ infinitely often, and in these cases, the number of samples received by $B$ is a polynomial, and hence $B$ breaks the hardness of LWE.

Next, we need to define the discrete Gaussian distribution:

Definition F.4 (Discrete Gaussian). Let $D_{\mathbb{Z}, r}$ denote the discrete Gaussian distribution over $\mathbb{Z}$, where the probability of $x$ is proportional to $e^{-\pi x^{2} / r^{2}}$.

We are now ready to state and prove Theorem 6.1:

Theorem 6.1. Let $\chi=D_{\mathbb{Z}, r}$, and $q \geq p \cdot \ell(C r \sqrt{n+\ell})^{\ell} n^{\omega(1)}$ for some suitable universal constant $C$. Let PRF be as in Construction 3, and suppose each $\mathbf{S}_{i}$ is drawn from $\chi^{n \times n}$. If the LWE problem is hard for modulus $q$ and distribution $\chi$, then PRF from Construction 3 is a QPRF.

Proof. The proof is very similar to that of Banerjee et al. Notice that our theorem requires $q \geq p \cdot \ell(C r \sqrt{n+\ell})^{\ell} n^{\omega(1)}$ whereas the original only requires $q \geq p \cdot \ell(C r \sqrt{n})^{\ell} n^{\omega(1)}$. We will explain 
why this is later. We first define a class of functions $G: \mathcal{K} \times[2]^{k} \rightarrow \mathbb{Z}_{q}^{m \times n}$ to be PRF without rounding. That is,

$$
G_{k}(x)=\mathbf{A}^{t} \prod_{i=1}^{\ell} \mathbf{S}_{i}^{x_{i}}
$$

Then $\operatorname{PRF}_{k}(x)=\left\lfloor G_{k}(x)\right\rceil_{p}$. We also define a related class of functions $\tilde{G}$ where $\tilde{G}=\tilde{G}^{(\ell)}$ and

- $\tilde{G}^{(0)}$ is a function from $[2]^{0}$ into $\mathbb{Z}_{q}^{m \times n}$ defined as follows: pick a random $\mathbf{A} \in \mathbb{Z}_{q}^{n \times m}$, and set $\tilde{G}^{(0)}(\epsilon)=\mathbf{A}^{t}$.

- $\tilde{G}^{(i)}$ is a function from $[2]^{i}$ into $\mathbb{Z}_{q}^{m \times n}$ defined as follows: pick a random $\tilde{G}^{(i-1)}$, pick $\mathbf{S}_{i} \leftarrow \chi^{n \times n}$ and for each $x^{\prime} \in[2]^{i-1}$, pick $\mathbf{E}_{x^{\prime}} \leftarrow \chi^{m \times n}$. Then

$$
\tilde{G}^{(i)}\left(x=x^{\prime} x_{i}\right)=\tilde{G}^{(i-1)}\left(x^{\prime}\right) \cdot \mathbf{S}_{i}^{x_{i}}+x_{i} \cdot \mathbf{E}_{x^{\prime}} \bmod q
$$

Let $A$ be an adversary that distinguishes PRF from a random function with probability $\epsilon$.

First, consider the case where $A$ sees a truly random function $U:[2]^{k} \rightarrow \mathbb{Z}_{p}^{m \times n}$. Replace $U$ with $\left\lfloor U^{\prime}\right\rceil_{p}$ where $U^{\prime}$ is a truly random function from $[2]^{k} \rightarrow \mathbb{Z}_{q}^{m \times n}$. For each input, the bias introduced by this rounding is negligible because $q \geq p n^{\omega(1)}$. Thus, by Theorem 1.1, the ability of $A$ to distinguish these two cases is negligible.

Now, let $B=\ell(C r \sqrt{n+k})^{\ell}$. Let $\operatorname{BAD}(y)$ be the event that

$$
\left\lfloor y+[-B, B]^{m \times n}\right\rceil_{p} \neq\left\{\lfloor y\rceil_{p}\right\}
$$

That is, $\operatorname{BAD}(y)$ is the event that $y$ is very close to another element in $\mathbb{Z}_{q}$ that rounds to a different value in $\mathbb{Z}_{p}$. Banerjee et al. show that for each $x$, the probability that $\operatorname{BAD}\left(U^{\prime}(x)\right)$ occurs is negligible. Therefore, according to Theorem 1.1, $\operatorname{BAD}\left(U^{\prime}(x)\right)$ as an oracle with outputs in \{True, False $\}$ is indistinguishable from the oracle that always outputs False. Hence, it is impossible for an algorithm making quantum queries to $U^{\prime}$ to find an $x$ such that $\operatorname{BAD}\left(U^{\prime}(x)\right)$ occurs, except with negligible probability.

The next step is to prove that $U^{\prime}$ and $\tilde{G}$ are oracle-indistinguishable. Once we have accomplished this, we replace $U^{\prime}$ with $\tilde{G}$. Then the probability that $A$ detects this change is negligible. Additionally, it is also impossible to find an $x$ such that $\operatorname{BAD}(\tilde{G}(x))$ occurs, except with negligible probability.

Lastly, we replace $\tilde{G}$ with $G$. Banerjee et al. show that as long as $\operatorname{BAD}(\tilde{G}(x))$ does not occur, $\left\lfloor\left.\tilde{G}(x)\right|_{p}=\left\lfloor G_{k}(x)\right\rceil_{p}=\operatorname{PRF}_{k}(x)\right.$ with all but negligible probability. Our modification to the parameters of the theorem (replacing $\sqrt{n}$ with $\sqrt{n+k}$ ) allows us to choose $C$ so that this probability is actually $2^{-\ell} \sigma$ for some negligible $\sigma$. Summing over all $2^{\ell}$ different $x$, we get that, except with negligible overall probability, $\operatorname{PRF}_{k}(x)=[\tilde{G}(x)]_{p}$ whenever $\operatorname{BAD}(\tilde{G}(x))$ does not occur.

Thus, if $A$ distinguishes $\operatorname{PRF}_{k}(x)$ from $[\tilde{G}(x)]_{p}$ with non-negligible probability, it must be that the sum over all queries made by $A$ of the sum of the query magnitudes of all the $x$ such that $\operatorname{BAD}(\tilde{G}(x))$ occurs is non-negligible (Theorems 3.1 and 3.3 of [BBBV97]). But this means we can find an $x$ such that BAD $(\tilde{G}(x))$ occurs with non-negligible probability (simply run $A$, and at a randomly chosen query, halt and sample the query). But, as we have already shown, this is impossible. 
Hence, we have shown that PRF is indistinguishable from a random function.

It remains to show that $U^{\prime}$ and $\tilde{G}$ are oracle-indistinguishable. We show that this is true given that the LWE problem is oracle-hard. Using Lemma F.3, we reach the same conclusion assuming LWE is hard, thus completing the theorem.

Let $B$ be an adversary that distinguishes $U^{\prime}$ from $\tilde{G}$ with probability $\epsilon$. Define hybrid $H_{i}$ as the case where $B$ is given the oracle $O_{i}$ where $O_{i}=\tilde{G}$, except that, in the recursive definition of $\tilde{G}, \tilde{G}^{(i)}$ is replaced with a truly random function. $H_{0}$ corresponds to the correct definition of $\tilde{G}$, and $H_{k}$ corresponds to $U^{\prime}$. Thus, there exists an $i$ such that $B$ distinguishes $H_{i}$ from $H_{i-1}$ with probability $\epsilon / \ell$.

Construct an adversary $C$ with access to an oracle $P:[2]^{i-1} \rightarrow \mathbb{Z}_{q}^{m \times n} \times \mathbb{Z}_{q}^{m \times n}$. $P$ is either a random function or each output is chosen according to the LWE distribution. In other words, $P(x)=\left(\mathbf{A}^{t}, \mathbf{B}^{t}\right)$, where either $\mathbf{A}(x)$ and $\mathbf{B}(x)$ are chosen at random for all $x$, or there is a secret $\mathbf{S} \leftarrow \chi^{n \times n}$ and $\mathbf{B}(x)^{t}=\mathbf{A}(x)^{t} \mathbf{S}+\mathbf{E}(x) \bmod q$ where $\mathbf{E}(x) \leftarrow \chi^{m \times n}$.

For each $j>i, C$ constructs random oracles $Q_{j}:[2]^{j-1} \rightarrow \mathbb{Z}^{m \times n}$ where $Q_{j}(x) \leftarrow \chi^{m \times n}$. $C$ also generates $\mathbf{S}_{j} \leftarrow \chi^{n \times n}$ for $j>i$. Then $C$ works as follows:

- Let $\tilde{G}^{(i)}\left(x=x^{\prime} x_{i}\right)= \begin{cases}\mathbf{A}\left(x^{\prime}\right)^{t} & \text { if } x_{i}=0 \\ \mathbf{B}\left(x^{\prime}\right)^{t} & \text { if } x_{i}=1\end{cases}$

- Let $\tilde{G}^{(j)}\left(x=x^{\prime} x_{j}\right)=\tilde{G}^{(j-1)}\left(x^{\prime}\right) \cdot \mathbf{S}_{j}^{x_{j}}+x_{j} \cdot Q_{j}\left(x^{\prime}\right) \bmod q$ for $j>i$.

- Let $O(x)=\tilde{G}^{(k)}(x)$

- Run $B$ with oracle $O$.

When $P$ is a random oracle, this corresponds to $H_{i}$. When $P$ is the LWE oracle, this corresponds to $H_{i-1}$. Thus, $C$ distinguishes these two cases with probability at least $\epsilon / \ell$. Under the assumption that LWE is oracle hard, this quantity, and hence $\epsilon$, are negligible. We then use Lemma F.3 to complete the theorem.

\section{References}

[BBBV97] Charles H. Bennett, Ethan Bernstein, Gilles Brassard, and Umesh Vazirani. Strengths and Weaknesses of Quantum Computing. SIAM Journal on Computing, 26:1510-1523, 1997.

$\left[\mathrm{BDF}^{+} 11\right]$ Dan Boneh, Özgür Dagdelen, Marc Fischlin, Anja Lehmann, Christian Schaffner, and Mark Zhandry. Random Oracles in a Quantum World. In Advances in Cryptology ASIACRYPT 2011, 2011.

[BHT97] Gilles Brassard, Peter Høyer, and Alain Tapp. Quantum Algorithm for the Collision Problem. ACM SIGACT News (Cryptology Column), 28:14-19, 1997.

[BL95] Dan Boneh and Richard J. Lipton. Quantum Cryptanalysis of Hidden Linear Functions. Advances in Cryptology - CRYPTO 1995, 1995. 
[BMR10] Dan Boneh, Hart Montgomery, and Ananth Raghunathan. Algebraic Pseudorandom Functions with Improved Efficiency from the Augmented Cascade. Proceedings of the 17th ACM Conference on Computer and Communications Security (CCS), pages 1-23, 2010 .

[BPR11] Abhishek Banerjee, Chris Peikert, and Alon Rosen. Pseudorandom Functions and Lattices. Advances in Cryptology - EUROCRYPT 2012, pages 1-26, 2011.

[DY05] Yevgeniy Dodis and Aleksandr Yampolskiy. A Verifiable Random Function with Short Proofs and Keys. Public Key Cryptography, 2005.

[GGM86] Oded Goldreich, Shafi Goldwasser, and Silvio Micali. How to Construct Random Functions. Journal of the ACM (JACM), 33(4):792-807, 1986.

[Gro96] Lov K. Grover. A Fast Quantum Mechanical Algorithm for Database Search. Proceedings of the 28th Annual ACM Symposium on Theory of Computing (STOC), pages 212-219, 1996.

[HILL99] Johan Håstad, Russell Impagliazzo, Leonid Levin, and Michael Luby. A pseudorandom generator from any one-way function. SIAM J. Comput., 28(4):1364-1396, 1999.

[LR88] Michael Luby and Charles Rackoff. How to construct pseudorandom permutations from pseudorandom functions. SIAM J. Comput., 17(2):373-386, April 1988.

[LW09] Allison B. Lewko and Brent Water. Efficient Pseudorandom Functions From the Decisional Linear Assumption and Weaker Variants. Proceedings of the 16th ACM Conference on Computer and Communications Security (CCS), pages 1-17, 2009.

[NC00] Michael A. Nielsen and Isaac Chuang. Quantum Computation and Quantum Information. American Journal of Physics, 70(5):558, 2000.

[NR95] Moni Naor and Omer Reingold. Synthesizers and Their Application to the Parallel Construction of Pseudo-Random Functions. Proceedings of the 36th IEEE Symposium on Foundations of Computer Science (FOCS), 1995.

[NR97] Moni Naor and Omer Reingold. Number-Theoretic Constructions of Efficient PseudoRandom Functions. Proceedings of the 38th IEEE Symposium on Foundations of Computer Science (FOCS), 51(2):231-262, 1997.

[NR99] Moni Naor and Omer Reingold. On the Construction of Pseudorandom Permutations : Luby-Rackoff Revisited. Journal of Cryptology, (356):29-66, 1999.

[NRR00] Moni Naor, Omer Reingold, and Alon Rosen. Pseudo-Random Functions and Factoring. Proceedings of the 32nd Annual ACM Symposium on the Theory of Computing (STOC), pages $1-23,2000$.

[Zha12] Mark Zhandry. Secure Identity-Based Encryption in the Quantum Random Oracle Model. In Advances in Cryptology - CRYPTO 2012, 2012. Full version available at the Cryptology ePrint Archives: http://eprint.iacr.org/2012/076/. 